Sociologie et sociétés

\title{
Transformations sociales et identités syndicales : l'institution syndicale à l'épreuve de la différenciation sociale contemporaine
}

\author{
Christian Lévesque, Gregor Murray et Stéphane Le Queux
}

Volume 30, numéro 2, automne 1998

Le syndicalisme

URI : https://id.erudit.org/iderudit/001431ar

DOI : https://doi.org/10.7202/001431ar

Aller au sommaire du numéro

\section{Éditeur(s)}

Les Presses de l'Université de Montréal

\section{ISSN}

0038-030X (imprimé)

1492-1375 (numérique)

Découvrir la revue

Citer cet article

Lévesque, C., Murray, G. \& Le Queux, S. (1998). Transformations sociales et identités syndicales : l'institution syndicale à l'épreuve de la différenciation sociale contemporaine. Sociologie et sociétés, 30(2), 131-154.

https://doi.org/10.7202/001431ar

\section{Résumé de l'article}

Deux hypothèses sur l'affaiblissement des identités syndicales sont soumises à une épreuve empirique à partir d'une étudeauprès des syndiqués d'une centrale syndicale au Québec (la Confédération des syndicats nationaux). La première insiste surla différenciation sociale ; la seconde sur l'effet structurant des conditions matérielles de travail et des stratégies des acteurs.Deux dimensions de l'identité syndicale sont étudiées : l'évaluation du caractère essentiel du syndicalisme et l'adhésion auxmodalités d'action les plus caractéristiques du syndicalisme industriel. Les diverses sources de différenciation sociale exercentpeu d'impact sur le caractère essentiel mais touchent lourdement l'adhésion aux modalités d'action. À l'encontre des conditionsmatérielles de travail, les stratégies des acteurs sont très importantes pour les deux dimensions. Les sources de différenciationsociale ne constituent pas une rupture par rapport au syndicalisme mais elles font appel à un renouveau de sesmodalités d'action. Ce renouveau doit partir d'une participation des personnes porteuses de ces nouveaux intérêts dans l'élaborationde nouvelles modalités d'action collectives.
Tous droits réservés @ C Les Presses de l'Université de Montréal, 1998

Ce document est protégé par la loi sur le droit d'auteur. L’utilisation des services d'Érudit (y compris la reproduction) est assujettie à sa politique d'utilisation que vous pouvez consulter en ligne.

https://apropos.erudit.org/fr/usagers/politique-dutilisation/ 


\section{Transformations sociales et identités syndicales : l'institution syndicale à l'épreuve de la différenciation sociale contemporaine $^{1}$}

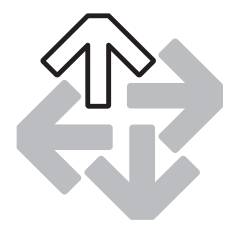

CHRISTIAN LÉVESQUE, GREGOR MURRAY et STÉPHANE LE QUEUX

Il est généralement admis que les lignes de différenciation sociale qui traversent la société contemporaine modifient profondément la dynamique syndicale. L'hypothèse sous-jacente est que les fondements des solidarités emblématiques de la formation des classes ouvrières, qui donnèrent lieu à l'émergence et à l'institutionnalisation des mouvements syndicaux de grande envergure, s'effriteraient devant la multiplication, l'affirmation et la «désimbrication » d'identités sociales dans une société de plus en plus souvent qualifiée de «postindustrielle » ou de «post-moderne ».

L'interrogation ici soulevée porte sur l'interface entre le syndicalisme, en tant qu'héritage institutionnel, et cette société postindustrielle qui serait à l'origine d'une remise en cause des modèles collectivistes sur lesquels reposent les identités syndicales. La différenciation croissante des rapports sociaux, qu'il s'agisse, par exemple, du mouvement vers des emplois à statut précaire ou de la scolarité accrue des salariés, serait responsable de l'anomie des formations collectives inhérentes au fait syndical; le syndicalisme se trouverait aujourd'hui en déficit de représentativité sociale (Hyman, 1997b).

Une autre lecture est toutefois possible. La dynamique syndicale serait alors appréciée en regard de l'exercice même de la représentation collective par rapport aux contextes particuliers dans lesquels elle s'exerce. Le sentiment d'appartenance au syndicat serait ainsi à considérer à la lumière des contextes matériels du travail où ces nouvelles sources de différenciation se manifestent. Dans la mesure où il y a affaiblissement du lien syndical, ce dernier peut-il être saisi indépendamment des milieux de travail et, en particulier, sans égard à la régulation plus ou moins médiate du rapport d'emploi opérée par le syndicat?

L'objectif de ce texte est de soumettre ces deux thèses à une épreuve empirique. Partant d'une étude des membres de la Confédération des syndicats nationaux au Québec, nous examinerons les

1. Cet article s'inscrit dans le cadre d'un projet de recherche sur les transformations de la représentation syndicale avec l'appui financier de la Confédération des syndicats nationaux (CSN), le Conseil de recherches en sciences humaines du Canada et le Fonds pour la formation de chercheurs et l'aide à la recherche. Outre les nombreuses personnes de la CSN qui ont collaboré à la réalisation de cette recherche, nous tenons à souligner la participation de Nicolas Roby. Nous remercions aussi les directrices de ce numéro thématique et deux évaluateurs anonymes pour leurs commentaires sur une version antérieure de ce texte. 
sources potentielles d'affaiblissement du sentiment d'appartenance des salariés en rapport avec un ensemble de traits culturels et de pratiques collectives qui caractérisent le syndicalisme ${ }^{2}$. Une fois la problématique approfondie et la méthode éclaircie, l'exercice qui suit se fera en deux temps qui couvrent chacun une dimension de l'identité syndicale. On examinera d'abord si les salariés jugent le syndicalisme essentiel. Si le salarié ne le considère pas comme «essentiel», son sentiment d'appartenance au syndicat sera sûrement moindre. Nous nous intéresserons ensuite à l'adhésion des salariés aux modalités d'action collective traditionnelles, par exemple la grève. L'expression de l'identité syndicale est ici plus particulière : il s'agit globalement de l'acceptation d'un ensemble de pratiques collectives qui a caractérisé le syndicalisme industriel.

En contexte nord-américain, où l'exclusivité de la représentation pour l'ensemble des salariés dans un lieu de travail donné est conférée à un seul syndicat ${ }^{3}$, l'identité syndicale peut couvrir un territoire considérable, passant du sentiment d'appartenance des salariés syndiqués les plus militants et engagés dans l'action syndicale aux salariés qui vraisemblablement manifestent peu d'intérêt à l'égard de l'institution syndicale et pour qui le sentiment d'appartenance serait très faible. Notre objet ici — l'identité syndicale, le lien syndical ou le rapport salarié-syndicat ${ }^{4}$ — est l'importance de ces différents sentiments d'appartenance au syndicat et, plus globalement, ce qu'il représente comme institution de régulation du travail.

Pour chacune des dimensions de l'identité syndicale, l'analyse s'exécutera en deux temps. D'abord, partant de la première thèse, il s'agira d'apprécier si les sources de différenciation sociale, qui caractérisent les sociétés contemporaines, provoquent un affaiblissement du sentiment d'appartenance au syndicat. Ensuite, s'inspirant de la seconde thèse, nous évaluerons dans quelle mesure les caractéristiques des contextes matériels de travail atténuent ou accentuent les identités syndicales.

\section{LES SOURCES D'AFFAIBLISSEMENT DES IDENTITÉS SYNDICALES}

Le syndicalisme connaît-il aujourd'hui une crise de «solidarité organique » ? De façon élémentaire, comme le dénote Hyman (1997b), sans différenciation, les solidarités seraient superflues. Or, celles-ci ne sont pas fixées a priori : le façonnement des solidarités syndicales s'appuie sur un travail d'organisation, mais également de délimitation et de légitimation de l'espace collectif (Offe et Wiesenthal, 1980). Le problème actuel ne relève-t-il pas dès lors de l'agrégation et de la formulation d'intérêts de plus en plus différenciés à l'intérieur de rapports de plus en plus individualisés (Hyman, 1997a ; Leisink et coll., 1996 ; Madsen, 1996) ?

La forme institutionnelle prédominante du syndicalisme, le syndicalisme industriel du milieu $\mathrm{du} \mathrm{Xx}^{\mathrm{e}}$ siècle, reposait sur une certaine homogénéité culturelle liée à la fabrication manufacturière par des travailleurs dont les parcours professionnels dépendaient surtout de l'intervention syndicale pour l'amélioration de leur sort (voir Murray, 1998). Pour reprendre l'analyse de Hobsbawn (1994, p. 306), ce qui distinguait les travailleurs de la classe ouvrière industrielle du vingtième siècle a été cet élément central de leurs vies — la collectivité — la prédominance du «nous » sur le « je ». Or, ce qui caractérise le changement social de l'ère contemporaine, c'est la radicalisation de la différenciation des rapports sociaux qui sous-tendent les collectivités syndicales jadis dominantes. Le marché du travail a connu dans la plupart des pays industrialisés de profonds bouleversements qui, à leur tour, ont été lourds de conséquences pour les organisations syndicales. Ces bouleversements

2. Nous cherchons ici à faire l'économie d'une riche littérature sur l'identité (voir Sainsaulieu, 1977; Dubar, 1991). Pour l'essentiel, l'identité renvoie à la façon dont l'individu se définit lui-même socialement par rapport à autrui. L'identité collective comprend le sentiment d'appartenance de l'individu au groupe auquel il s'identifie. Par extension, l'identité syndicale renvoie au sentiment d'appartenance à l'ensemble des pratiques qui caractérisent le syndicalisme.

3. Dans un milieu de travail où la majorité de salariés choisissent un régime de représentation syndicale, un salarié est ainsi syndiqué dès que la majorité de ses collègues ont opté, à un moment donné, pour ce régime de représentation collective et n'ont pas choisi, depuis lors, d'y renoncer.

4. Expression d'une équivalence générale dans les régimes de l'Europe du Nord et les pays «anglo-saxons », typiquement le syndicat couvre la quasi-totalité de salariés dans un même milieu de travail. 
conduisent à une différenciation très accentuée, voire à des déchirements du tissu de la collectivité ouvrière jusque-là dominante. Ce processus de différenciation s'échelonne sur une longue période, mais ses manifestations sont devenues beaucoup plus apparentes avec les crises économiques successives des années 1970 et 1980.

Les interprétations des observateurs scientifiques sur les recompositions socioprofessionnelles en cours et leurs implications pour les mouvements syndicaux contemporains concordent relativement avec ce propos (même si elles ne font pas toujours l'objet d'une démonstration empirique). Valkenburg (1996, p. 90) évoque l'érosion des cadres de référence collectifs fondés sur la société industrielle, sur lesquels l'action des syndicats industriels reposait, à la faveur des «projets » individuels et évolutifs. Müller-Jentsch (1988) identifie trois sources de crise du syndicalisme : l'hétérogénéité croissante de la force de travail, qui induit une crise d'agrégation des intérêts ; la décentralisation des régimes de régulation au niveau de l'entreprise, qui alimente une crise de « loyauté » des syndiqués ; l'incapacité relative des syndicats à couvrir les nouveaux segments de la force de travail dans les secteurs en croissance. Hyman (1997b, p. 521) fait également ressortir différents facteurs de crise des projets collectivistes parmi lesquels on retrouve : une accélération de la différenciation interne de la main-d'œuvre ; une intensification de la concurrence, des restructurations et une dérégulation conduisant à des luttes intra-classes et à la préférence pour des solutions micro-sociales ; enfin, une érosion des idéaux collectivistes au sein même des mouvements syndicaux, symptôme d'une absence de projet alternatif. Le BIT (1997, p. 21) relève les difficultés des fédérations syndicales d'intégrer « cette diversité croissante des priorités de leurs membres » : « les préoccupations paraissent aujourd'hui plus variées que précédemment selon le secteur d'activité, la profession, les qualifications, l'âge ou le sexe ».

Plusieurs facteurs peuvent ainsi concourir à l'affaiblissement du lien syndical. Nous nous arrêterons ici sur les conséquences de six lignes de différenciation : la composition sexuelle de la main-d'œuvre, la scolarité des salariés, les changements culturels qui se manifestent par des mutations de valeurs entre générations, les mouvements vers des emplois dans les services et dans les établissements de petite taille et vers des emplois à statut précaire.

La féminisation de la force de travail a constamment marqué les économies industrielles au cours des dernières décennies (voir Jenson et coll., 1988). Le travail des femmes n'est pas à l'image de l'emploi des hommes. On y relève des salaires inférieurs, une forte ségrégation professionnelle, des statuts souvent plus précaires et, très souvent, une durée du travail moindre... À cette spécificité professionnelle du travail des femmes s'ajoutent des facteurs qui relèvent des modes de reproduction sociale, notamment le soin des enfants et le travail domestique non salarié, dont la responsabilité première incombe le plus souvent aux femmes. Ces facteurs, relevant tant de la production que de la reproduction sociale, affaibliraient l'identité syndicale ${ }^{5}$.

La scolarité accrue des salariés constitue une deuxième source de différenciation sociale. Heckscher (1988, p. 67) note que les salariés ayant une formation supérieure n'épousent pas les formes d'action les plus caractéristiques du syndicalisme industriel, dont l'opposition axiomatique aux orientations patronales et le rattachement à une classe sociale distincte. Ils tendent plutôt fortement à la mobilité et au développement professionnels ${ }^{6}$. Valkenburg (1996, p. 91) insiste plus particulièrement sur la nouvelle importance de la connaissance dans l'élaboration de projets individuels de travailleurs plus scolarisés et des identités sociales qui s’y rattachent. Il y a donc, au minimum,

5. D'autant plus que, malgré une certaine «féminisation » du discours syndical, plusieurs auteurs font état du peu de progrès réel dans les pratiques syndicales qui touchent la ségrégation professionnelle, ou encore les idéologies patriarcales qui tendent à « marginaliser» les préoccupations des femmes. Voir Briskin et McDermott (1993).

6. Dans leur analyse des implications des nouvelles formes d'organisation de la production, Kern et Sabel (1992, p. 228) avancent aussi la possibilité d'un certain affaiblissement du lien syndical dans les groupes de travailleurs les plus scolarisés dans les industries de fabrication en Allemagne. Des modifications dans le parcours professionnel de ces travailleurs, suggèrent-ils, notamment la plus grande importance de l'éducation universitaire à l'extérieur des lieux de travail, impliquent qu'ils consacrent moins de temps à se former sur le «plancher» et qu'ils ont «less time to form loyalties to labour». 
risque de désinvestissement à l'égard des valeurs collectives qui caractérisaient le modèle prédominant du syndicalisme industriel.

Un rapport récent du Bureau international du travail (1997, p. 20) sur le syndicalisme souligne un problème particulier chez les jeunes, «manifestement moins attirés que leurs aînés [...] par le mouvement syndical ». Plusieurs motifs sont avancés pour expliquer cet affaiblissement du lien syndical chez les jeunes : les problèmes d'accès à l'emploi et le caractère épisodique de leurs expériences professionnelles; des changements de valeurs sociales par rapport à leurs parents ; leur plus grande sensibilité aux nouvelles formes de mobilisation du personnel qui mettent l'accent sur la responsabilité individuelle. Un ensemble de facteurs, souvent contradictoires, diminue ainsi la pertinence de l'institution syndicale pour les jeunes.

La faible pénétration syndicale dans le secteur des services privés est notoire (voir Bergeron, 1994 ; Murray, 1994). Outre la taille restreinte de beaucoup d'établissements et l'importance relative de nouvelles catégories de salariés et d'emplois, facteurs que nous abordons distinctement, la mobilité professionnelle accentuée des travailleurs dans ce secteur et la nature du travail soulèvent de nouveaux défis pour la représentation syndicale. Par sa conception figée du syndicat majoritaire dans une entreprise morcelée, le régime nord-américain de représentation collective n'arrive pas à suivre le parcours professionnel des salariés dans ce secteur. Les emplois dans les services se distinguent aussi par le caractère relationnel de l'accomplissement du travail par des prestataires de services, dimension généralement absente dans le secteur des biens. Les modalités traditionnelles d'action collective peuvent paraître moins appropriées, la grève touchant directement le consommateur des services?

Le déclin relatif du gigantesque établissement industriel — l'archétype du collectif de travail ouvrier et du syndicalisme industriel — et la croissance considérable de l'emploi dans les petits établissements, y compris dans les réseaux productifs autour d'un grand établissement, constituent une autre source de différenciation sociale. Plusieurs auteurs ont déjà suggéré que les relations plus personnalisées entre employés et employeur dans la petite entreprise rendent moins pertinente la représentation syndicale (Dion et Hébert, 1989, p. 12). Les syndicats dans les petits établissements se heurtent aussi à un problème de ressources : ressources matérielles, bien sûr, dans la capacité à organiser des services (voir Brassard et Murray, 1997), mais aussi ressources idéologiques ou discursives. En particulier, ils éprouveraient de la difficulté à mobiliser un contre-projet, se trouvant dès lors confinés dans une «logique sociale réactive» (Tixier, 1992, p. 59).

Enfin, dans une perspective de réduction des coûts salariaux et d'augmentation de la flexibilité organisationnelle, et en raison de l'abondance de la main-d'œuvre en période de chômage élevé, les employeurs ont recours à une prolifération de formes d'emploi autres que le modèle traditionnel, c'est-à-dire l'emploi régulier à temps complet. Cette différenciation grandissante des statuts, cet écart croissant entre insiders et outsiders, représente une autre source de différenciation sociale. Non seulement l'emploi précaire est moins syndiqué que le travail régulier à temps complet, mais ces nouveaux groupes de salariés participent moins aux activités syndicales (Paquet, 1996 ; Schetagne, 1997 ; Sinclair, 1996).

On suggère généralement que ces changements réduisent la capacité des organisations syndicales d'élaborer et de soutenir une communauté d'intérêts ${ }^{8}$. Le syndicalisme est donc amené à reconsidérer ses bases identitaires traditionnelles, dont le travailleur industriel constitue le modèle. Pour reprendre les propos de Hyman (1994, p. 118), ces changements posent problème en regard de la volonté des salariés de se syndiquer et de maintenir leur adhésion, des attentes des syndiqués vis-

7. Comme l'observe Hyman (1994, p. 118), dans le cas des services publics, non seulement les salariés font face à des coupures budgétaires et à une diminution des services, mais leur capacité de mobilisation est réduite car la grève affecte aussi bien le consommateur que l'employeur et peut, par conséquent, aliéner l'appui populaire.

8. On pourrait, bien sûr, ajouter d'autres changements importants, dont l'organisation de la production (Tixier, 1992, pp. 113-136), ou encore la mondialisation des rapports économiques (Hyman, 1997b), mais notre présentation de cette première thèse se limite aux modifications les plus caractéristiques de l'évolution contemporaine des marchés du travail. 
à-vis leurs syndicats, du lien entre le rapport au syndicat et les autres identités au travail et hors travail, de la participation à la vie syndicale et de la volonté de poursuivre ses politiques.

On peut proposer une tout autre lecture de l'évolution contemporaine de l'identité sociale. Au minimum, tout en concédant l'intérêt de ces nouvelles sources de différenciation, on peut se demander si on ne peut pas saisir leur effet indépendamment des contextes de travail dans lesquels elles se manifestent. Plus radicalement, ces contextes de travail ne structurent-ils pas directement, en premier lieu, le lien syndical ? La différenciation du salariat n'est guère nouvelle ; même le prolétariat industriel s'est constitué graduellement sur la base d'habiletés différenciées, de communautés ethniques, de spécificités locales et de régionalismes de toutes sortes. Autrement dit, une nouvelle hypothèse postulerait que tout affaiblissement du lien syndical n'est pas le résultat de nouvelles sources de différenciation, mais plus directement des contextes de travail. La matérialité du rapport d'emploi, largement entendue, serait ainsi structurante, dans la mesure où l'identité syndicale se constitue dans ce contexte. On peut en évoquer au moins deux dimensions pertinentes : les conditions matérielles de travail et les relations sociales de production.

La littérature syndicale anglo-américaine laisse une large place à l' « instrumentalité » dans le rapport au syndicat, qu'il s'agisse de la volonté du salarié de se syndiquer (la propension syndicale), de l'engagement du syndiqué à l'égard de son organisation (union commitment) ou de la satisfaction générale à l'égard du syndicat. En ce qui concerne la propension syndicale, l'intérêt premier pour la syndicalisation part d'un constat d'insatisfaction au travail (voir Wheeler et McClendon, 1991). On ne peut ainsi comprendre le lien au syndicat sans renvoyer aux conditions de travail et, plus particulièrement, à l'évaluation du caractère instrumental du syndicat dans l'amélioration de ces conditions. Barling et coll. (1992), qui recensent plusieurs dimensions du rapport au syndicat, identifient l'insatisfaction au travail et l'évaluation du caractère instrumental du syndicat comme les facteurs qui jouent le plus dans la détermination de la propension à se syndiquer. Guest et Dewe (1988), dans une étude réalisée dans l'industrie électronique en Grande-Bretagne, arrivent à la même conclusion en ce qui a trait à l'engagement syndical. Enfin, dans une étude sur le degré de satisfaction des salariés envers leur syndicat, Fiorito et coll. (1988) notent l'importance des enjeux regroupés sous l'étiquette bread and butter par rapport à ceux liés à la qualité de vie au travail. Bref, pour renouer avec l'étude de la conscience ouvrière de Perlman (1949) en contexte américain, une analyse de l'affaiblissement du lien au syndicat ne peut faire l'économie de l'évaluation que font les membres de leurs conditions matérielles de travail et de l'impact du syndicat sur ces conditions.

Les relations sociales de production représentent une autre dimension du contexte de travail. La spécificité du rapport d'emploi tient à la subordination juridique et sociale qu'implique le rapport salarié mais aussi, selon cette analyse, à l'organisation sociale nécessaire à l'extraction de l'effort par l'employeur (voir en particulier Edwards, 1986). La qualité des relations sociales de production, et en particulier, le comportement de l'employeur exerceraient une influence déterminante sur l'identité syndicale. Dans une étude auprès de salariés dans six communautés britanniques, Gallie (1996) cherche à expliquer le maintien de l'engagement syndical dans un contexte de récession économique. Il souligne l'importance des variables structurelles, en particulier l'attitude de l'employeur dans le maintien du lien syndical. La présence d'un employeur hostile aux syndicats entraînerait une moins forte adhésion des salariés à l'institution syndicale. Ce résultat rejoint largement d'autres études, notamment américaines, sur l'effet dissuasif des stratégies privilégiées par des employeurs pour éviter la présence syndicale. Si le comportement de l'employeur peut infléchir l'identité syndicale, il en va de même pour le comportement du syndicat. Fiorito et coll. (1988) montrent que la qualité des relations entre les membres et leur syndicat a un effet structurant sur l'évaluation que les membres font de leur syndicat. Newton et Mcfarlane Shore (1992, p. 289) notent que la communication de l'information aux nouveaux membres et la qualité de l'information sur la négociation collective peuvent renforcer l'engagement syndical. Ces travaux confortent, par ailleurs, l'idée voulant qu'il incombe aux organisations syndicales de redéfinir les identités collectives pour mieux tenir compte des nouvelles sources de différenciation sociale. 
Cette seconde thèse insiste davantage sur l'effet structurant tant de la matérialité du rapport d'emploi que du comportement des acteurs sur l'identité syndicale. Elle cherche à cerner les variations dans l'identité syndicale à partir de la représentation que se font les acteurs de leurs conditions de travail et des relations qui se développent dans leur lieu de travail.

Deux thèses, à la fois opposées et complémentaires, seront ainsi examinées pour rendre compte des variations de l'identité syndicale. La première met l'accent sur des facteurs extragènes à l'acteur syndical ; la seconde centre davantage l'analyse sur le contexte matériel dans lequel les salariés se constituent en collectivités de travail. Ni l'une ni l'autre de ces thèses n'a fait l'objet d'une analyse empirique systématique. Notre objectif consiste à les évaluer et à mieux comprendre comment, une fois conjuguées l'une à l'autre, elles façonnent les identités syndicales. La validité et la pertinence relative de ces thèses seront considérées en regard de deux dimensions constitutives de l'identité syndicale : le caractère essentiel de l'action syndicale et l'adhésion des salariés aux modalités d'action collective traditionnelles.

\section{LES PRÉALABLES MÉTHODOLOGIQUES}

Pour vérifier la validité de ces deux thèses, nous prenons appui sur une étude réalisée en 1994-1995 en collaboration avec la Confédération des syndicats nationaux (CSN) auprès d'un échantillon de responsables de syndicats locaux et de membres issus de ces syndicats. Dans un premier temps, un questionnaire a été posté à l'ensemble des syndicats. Au total, 977 responsables d'autant de syndicats ont complété le questionnaire, ce qui correspond à un taux de réponse de 48,6\%. Dans cette banque de données, nous avons sélectionné au hasard 308 unités syndicales en fonction de leur taille et de leur localisation sectorielle. Nous avons, dans un deuxième temps, constitué, de façon aléatoire, un échantillon de 2288 personnes. De ce nombre, 1457 ont complété un entretien téléphonique d'une durée moyenne de 22,5 minutes, ce qui correspond à un taux de réponse de $63,9 \% 9$.

Les membres interviewés sont donc issus de 308 unités syndicales, dont 24,1\% sont des syndicats du secteur de la fabrication des biens, 25,6\% sont des syndicats des services privés et 50,3\% sont des syndicats des services publics. En ce qui concerne la répartition des membres, $21,6 \%$ se situent dans le secteur des biens, $15,7 \%$ dans le secteur des services privés et $62,7 \%$ dans le secteur public. La répartition des membres selon la taille du syndicat se lit comme suit : $14,5 \%$ se retrouvent dans des syndicats de moins de 50 membres, 39,9\% dans des syndicats de 50 à 249 membres, puis $45,6 \%$ dans des syndicats de 250 membres et plus. La répartition de cet échantillon selon la localisation sectorielle et la taille des unités correspond à l'ensemble des membres de la CSN. Autrement dit, sur la base de ces deux critères, nous avons constitué un échantillon représentatif du membership de la CSN.

Compte tenu de la forte concentration des membres de la CSN dans les services, notamment dans les services publics, on y retrouve un fort contingent de femmes $(55,2 \%)$ et une forte proportion de membres ayant un emploi non standard $(31,7 \%)^{10}$. D'ailleurs, les caractéristiques socioprofessionnelles des membres sont intimement reliées à leur localisation sectorielle. Dans le secteur des biens, la main-d'œuvre est majoritairement masculine $(87,0 \%)$, dispose d'un emploi régulier à temps complet $(92,7 \%)$ et a une formation de niveau secondaire ou moins $(78,3 \%)$. À l'inverse, dans le secteur public, la main-d'œuvre se compose majoritairement de femmes $(70,0 \%)$ ayant soit une formation collégiale $(30,6 \%)$, soit une formation universitaire $(25,6 \%)$. Dans ce secteur, $40,0 \%$ des membres occupent un emploi non standard. Le portrait dans les services privés est plus

9. Les entrevues téléphoniques ont été réalisées par la compagnie de sondage CROP dans un intervalle de deux semaines, du 8 au 20 décembre 1995. Parmi les non-répondants, 17,8\% n'ont pu être rejoints, 16,9\% ont refusé de participer (l'individu ou le ménage) et $1,6 \%$ ont abandonné en cours d'entrevue.

10. Pour les fins de cet article, nous établissons une distinction entre les membres qui ont un emploi standard, c'està-dire un emploi régulier à temps complet, et ceux qui ont un emploi non standard, à savoir les emplois à temps partiel, temporaires, saisonniers, sur appel, occasionnels ou contractuels. 
contrasté : 54,1\% des membres sont des femmes, $68,1 \%$ y ont un emploi à temps complet régulier et une forte proportion d'entre eux ont une formation collégiale $(33,2 \%)$ ou universitaire $(14,0 \%)$. Il faudra nécessairement tenir compte de ces interrelations lors de l'interprétation des données.

L'intérêt particulier de notre démarche méthodologique tient à notre capacité à caractériser le contexte matériel de travail à partir de données provenant des membres et des responsables de syndicats locaux. Trois catégories de variables sont privilégiées pour décrire ces contextes. La première renvoie à l'évaluation que font les salariés de leurs conditions de travail. Trois indicateurs sont ici retenus : leur évaluation de l'évolution de leur sécurité d'emploi et de leur charge de travail au cours des trois dernières années et leur satisfaction à l'égard de leur travail ${ }^{11}$.

La deuxième catégorie de variables cherche à cerner le comportement de l'employeur. De nouveau, trois indicateurs sont privilégiés. Le premier provient des données collectées auprès des membres. Il s'agit de leur perception du comportement de l'employeur vis-à-vis du syndicat : le considère-t-il ou non comme un adversaire? Les deux autres sont issus des données obtenues auprès des responsables des syndicats locaux. D'une part, ils devaient nous indiquer jusqu'à quel point la direction de l'établissement cherche à contourner la convention collective. D'autre part, afin de déterminer si l'employeur tente de créer un climat d'intimidation et de peur, nous leur avons demandé si les membres de leur syndicat évitaient de militer parce qu'ils craignaient la réaction de l'employeur.

La dernière catégorie de variables tente de cerner le fonctionnement et la position du syndicat local. Nous avons ainsi posé aux membres deux questions qui permettent de décrire le fonctionnement démocratique du syndicat : «Le syndicat tient-il compte de l'opinion des membres ? « Le syndicat explique-t-il clairement ses décisions ? » Nous avons aussi obtenu des informations auprès des responsables sur la position du syndicat sur les changements apportés à l'organisation du travail. De ces données, on peut distinguer trois grandes positions qui ne sont pas mutuellement exclusives : une position d'appui (le syndicat travaille conjointement avec l'employeur pour résoudre les problèmes d'organisation du travail) ; une position d'opposition (le syndicat s'oppose car les changements vont à l'encontre de l'intérêt des membres) ; une position pro-active (le syndicat exerce des pressions sur l'employeur pour modifier l'organisation du travail).

\section{LE CARACTÈRE ESSENTIEL DU SYNDICAT}

La première dimension constitutive de l'identité syndicale concerne la pertinence même de la présence syndicale aux yeux des membres, autrement dit le caractère essentiel de l'action syndicale. Cette dimension recouvre un des axes centraux privilégiés pour cerner l'engagement syndical, à savoir la loyauté envers le syndicalisme ${ }^{12}$. Selon Klandermans (1989), la loyauté envers le syndicalisme implique au minimum le désir de demeurer syndiqué. Pour capter cette dimension, nous avons posé aux membres la question suivante : «Si vous aviez le choix, préféreriez-vous être syndiqué ou ne pas l'être ? » À cette question, 85,7\% des membres ont répondu par l'affirmative, 11,5\% par la négative et un faible pourcentage n'étaient pas en mesure de se prononcer $(2,8 \%)$. De manière à obtenir une évaluation plus générique, nous leur avons aussi demandé jusqu'à quel point ils étaient en accord avec la proposition suivante : «Les syndicats sont essentiels pour défendre les conditions de travail des

11. Sur une échelle variant de un à quatre, les membres devaient nous indiquer leur degré de satisfaction vis-à-vis de leur rémunération, leur sécurité d'emploi, l'influence qu'ils exercent sur leur travail, leurs possibilités de se réaliser dans leur travail et leurs perspectives de promotion. En raison de la forte corrélation entre chacun de ces indices de satisfaction, un indice général a été construit. Cet indice permet de distinguer les membres qui sont satisfaits de leur travail de ceux qui se disent insatisfaits.

12. Suite aux travaux de Gordon et coll. (1980), plusieurs auteurs américains et européens se sont intéressés à la question de l'engagement syndical (pour une revue des recherches américaines sur le union commitment, voir Barling, Fullagar et Kelloway, 1992). Quatre dimensions sont généralement retenues pour cerner le union commitment : une attitude de loyauté et un sentiment de responsabilité à l'égard de son syndicat, la disposition à s'impliquer pour celui-ci et la foi dans le syndicalisme. 
travailleurs. » À cette proposition, 62,6\% ont affirmé qu'ils étaient tout à fait d'accord, 24,2\% qu'ils étaient plutôt d'accord et $13,2 \%$ se sont dit plutôt ou tout à fait en désaccord.

Un premier constat s'impose : les thèses suggérant un affaiblissement du lien syndical, notamment en ce qui a trait à la pertinence des syndicats en tant qu'institution représentative, sont loin d'être confirmées par nos données. Une forte majorité de personnes préfèrent être syndiquées et considèrent que les syndicats sont essentiels pour défendre les conditions de travail. Sans remettre en cause ce constat général, il faut reconnaître que l'opinion des membres n'est pas complètement homogène ; c'est précisément cette variation qui nous intéresse ici. En combinant ces deux questions, trois cas de figure se présentent. Le premier regroupe les membres qui considèrent le syndicalisme comme essentiel $(62,6 \%)$ : ils préfèrent être syndiqués et n'hésitent pas à soutenir que les syndicats sont essentiels pour défendre les conditions de travail des salariés. Le deuxième cas de figure comprend les membres estimant que le syndicalisme est peu essentiel (13,2\%), soit qu'ils préfèrent ne pas être syndiqués, soit qu'ils ne soient pas en accord avec la proposition voulant que les syndicats soient essentiels pour défendre les conditions de travail. Le troisième cas regroupe des membres qui se situent entre ces deux extrêmes $(24,2 \%)$ : ils préfèrent être syndiqués mais ne sont pas tout à fait convaincus (plutôt d'accord) que les syndicats sont essentiels pour défendre les conditions de travail des salariés.

Comme on peut le constater à la lecture du tableau 1, cette première dimension du lien au syndicat varie significativement selon le sexe, la scolarité, l'âge, le statut d'emploi et le secteur d'activités. Une lecture rapide de ce tableau suggère que les femmes, les jeunes (moins de 30 ans), les membres ayant une formation post-secondaire, ceux ayant un emploi non standard tout comme ceux qui travaillent dans le secteur public sont moins portés à croire les syndicats essentiels. On notera en particulier que $15,6 \%$ des femmes considèrent le syndicat peu essentiel, pourcentage qui chute à 10,3\% chez les hommes. La cohorte d'âge des moins de 30 ans renferme aussi une plus forte proportion de membres estimant que le syndicat est peu essentiel : ils sont un peu plus de $18 \%$ à croire qu'il est peu essentiel et seulement 51,1\% à soutenir l'inverse. De la même manière, les personnes occupant un emploi non standard et celles ayant une formation collégiale sont moins portées à juger le syndicat essentiel : 15,2\% des membres avec une formation collégiale et 16,8\% des membres ayant un emploi non standard jugent le syndicat peu essentiel, alors que ce pourcentage chute respectivement à $12,2 \%$ et $10,3 \%$ parmi les membres ayant une formation de niveau secondaire et un emploi régulier à temps complet. Il en résulte que l'appréciation des membres quant à l'importance du syndicat est plus mitigée dans le secteur des services, en particulier dans les services publics : 59,6\% d'entre eux jugent le syndicat essentiel et 14,6\% l'estiment peu essentiel.

Cette analyse préliminaire des données laisse présager un certain affaiblissement, certes limité mais néanmoins réel, du lien salarié-syndicat chez les femmes, les jeunes, les membres plus scolarisés, les membres ayant un emploi non standard et ceux dans le secteur public. Bref, à première vue, la thèse de la différenciation sociale semble confirmée, Avant de tirer une telle conclusion, examinons si l'appréciation du caractère essentiel du syndicalisme varie selon le contexte matériel dans lequel les salariés se constituent en collectivités de travail.

On remarquera à la lecture du tableau 2 que l'appréciation du caractère essentiel du syndicalisme ne varie pas en fonction de l'évaluation que font les membres de leurs conditions de travail. Ni leur degré de satisfaction à l'égard de leur travail, ni l'ampleur des pressions sur l'emploi et la charge de travail ne sont associés aux variations dans l'évaluation du caractère essentiel du syndicalisme. Le comportement de l'employeur vis-à-vis du syndicat (perçoit-il le syndicat comme adversaire ou non) et la façon dont il administre la convention collective ne sont pas non plus directement associés à l'importance accordée par les membres au syndicalisme. Cependant, dans un contexte où, selon les représentants syndicaux, l'employeur crée un climat d'intimidation, les membres sont moins susceptibles de juger le syndicat essentiel $(59,2 \%$ contre $64,0 \%)$ et plus portés à le considérer peu essentiel $(17,6 \%$ versus $11,4 \%)$. Des trois types de variables retenues pour décrire le contexte, le comportement du syndicat se révèle le plus significatif. Ce ne sont pas tant les variables caractérisant sa position en matière d'organisation du travail comme celles désignant son fonction- 
TABLEAU 1

Évaluation des membres du caractère essentiel du syndicat selon les sources de différenciation sociale

\begin{tabular}{|c|c|c|c|}
\hline & Essentiel & Plutôt essentiel & Peu essentiel \\
\hline \multicolumn{4}{|l|}{ Sexe $^{* *}$} \\
\hline Homme $(n=652)$ & 66,3 & 23,5 & 10,3 \\
\hline Femme $(\mathrm{n}=795)$ & 59,6 & 24,8 & 15,6 \\
\hline \multicolumn{4}{|l|}{ Âge* } \\
\hline Moins de 30 ans $(n=172)$ & 51,7 & 29,7 & 18,6 \\
\hline Entre 30 et 39 ans $(n=510)$ & 61,6 & 25,9 & 12,5 \\
\hline Entre 40 et 49 ans $(n=504)$ & 64,7 & 22,2 & 13,1 \\
\hline Plus de 50 ans $(n=261)$ & 67,8 & 21,1 & 11,1 \\
\hline \multicolumn{4}{|l|}{ Scolarité* } \\
\hline Secondaire ou moins $(n=763)$ & 66,4 & 21,4 & 12,2 \\
\hline Formation collégiale $(n=409)$ & 57,2 & 27,6 & 15,2 \\
\hline Formation universitaire $(\mathrm{n}=274)$ & 60,2 & 26,6 & 13,1 \\
\hline \multicolumn{4}{|l|}{ Statut d'emploi $* *$} \\
\hline Temps complet régulier $(\mathrm{n}=994)$ & 65,2 & 23,3 & 11,5 \\
\hline Autres $(n=452)$ & 57,1 & 26,1 & 16,8 \\
\hline \multicolumn{4}{|l|}{ Secteur d'activité* } \\
\hline Biens $(n=315)$ & 70,8 & 19,0 & 10,2 \\
\hline Services privés $(n=227)$ & 63,4 & 24,7 & 11,9 \\
\hline Services publics $(n=905)$ & 59,6 & 25,9 & 14,6 \\
\hline \multicolumn{4}{|l|}{ Taille du syndicat } \\
\hline Petit $(<50)(\mathrm{n}=210)$ & 66,2 & 20,5 & 13,3 \\
\hline Moyen $(50-249)(\mathrm{n}=577)$ & 62,9 & 23,1 & 14,0 \\
\hline Grand $(>250)(\mathrm{n}=660)$ & 61,2 & 26,4 & 12,4 \\
\hline
\end{tabular}

$* \mathrm{p}<, 05 ; * * \mathrm{p}<, 005$

nement démocratique qui interviennent dans l'évaluation du caractère essentiel du syndicalisme. Lorsqu'un membre juge que le syndicat ne tient pas compte de l'opinion des membres ou qu'il n'explique pas clairement ses positions, il a tendance à considérer le syndicat peu essentiel. À titre d'exemple, parmi les membres évaluant que le syndicat ne tient pas compte de leur opinion, 31,9\% estiment le syndicat peu essentiel, pourcentage qui chute à $8,6 \%$ chez les membres jugeant que le syndicat tient compte de l'opinion des membres.

L'évaluation du caractère essentiel du syndicalisme en tant qu'institution représentative semble donc fluctuer à la fois selon le contexte matériel et les sources de différenciation sociale. Il s'agit maintenant de cerner l'importance relative de l'ensemble de ces variables et d'examiner de quelles façons elles se conjuguent les unes aux autres en les prenant simultanément en considération dans une même analys $\mathrm{s}^{13}$. Les résultats des analyses multivariées sont exposés dans le tableau 3 , où on distingue les variables permettant de départager les membres qui considèrent le syndicalisme peu essentiel de ceux qui le jugent essentiel (colonne 1), les membres le croyant peu essentiel de ceux qui le jugent plutôt essentiel (colonne 2) et les membres le considérant plutôt essentiel de ceux l'estimant essentiel (colonne 3).

De l'ensemble des variables retenues, sept permettent de distinguer les membres qui estiment le syndicat peu essentiel de ceux le jugeant essentiel (colonne 1). Ces variables se regroupent en

13. Compte tenu de la composition de la variable dépendante (trois valeurs), la régression logistique a été retenue pour identifier les variables permettant de différencier les trois groupes en opposant de façon successive deux groupes (peu essentiel contre essentiel, peu essentiel contre plutôt essentiel et plutôt essentiel contre essentiel). Toutes les variables indépendantes ont été dichotomisées de façon à mesurer leur capacité explicative (odds ratio). Cette façon de procéder, que nous empruntons à Gallie (1996, p. 167), permet d'établir les probabilités associées à chacune des variables. Soulignons également qu'en raison de la forte corrélation entre le sexe du répondant et sa localisation sectorielle, ces deux variables ont été combinées l'une à l'autre, ce qui permet de distinguer cinq catégories : les hommes dans le secteur des biens, les femmes dans les services privés, les hommes dans les services privés, les femmes dans les services publics et les hommes dans les services publics. 
TABLEAU 2

Évaluation des membres du caractère essentiel du syndicat selon les variables caractérisant le contexte matériel de travail

\begin{tabular}{|c|c|c|c|}
\hline & Essentiel & Plutôt essentiel & Peu essentiel \\
\hline \multicolumn{4}{|l|}{ Conditions de travail } \\
\hline \multicolumn{4}{|l|}{ Satisfaction au travail } \\
\hline Élevée $(\mathrm{n}=756)$ & 63,1 & 25,3 & 11,6 \\
\hline Faible $(n=691)$ & 62,1 & 23,0 & 14,9 \\
\hline \multicolumn{4}{|l|}{ Sécurité d'emploi } \\
\hline Maintenue $(\mathrm{n}=1088)$ & 63,2 & 24,3 & 12,5 \\
\hline Diminuée $(\mathrm{n}=353)$ & 60,6 & 24,1 & 15,3 \\
\hline \multicolumn{4}{|l|}{ Charge de travail } \\
\hline Maintenue $(\mathrm{n}=566)$ & 62,2 & 25,4 & 12,4 \\
\hline Augmentée $(\mathrm{n}=873)$ & 62,8 & 23,4 & 13,9 \\
\hline \multicolumn{4}{|l|}{ Employeur } \\
\hline \multicolumn{4}{|l|}{ Se comporte en adversaire } \\
\hline Non $(\mathrm{n}=802)$ & 62,6 & 25,8 & 11,6 \\
\hline Oui $(n=645)$ & 62,6 & 22,2 & 15,2 \\
\hline \multicolumn{4}{|c|}{ Cherche à contourner la convention collective } \\
\hline Non $(n=663)$ & 61,2 & 24,1 & 14,6 \\
\hline Oui $(\mathrm{n}=773)$ & 63,9 & 24,2 & 11,9 \\
\hline \multicolumn{4}{|c|}{ Crée un climat d'intimidation* } \\
\hline Oui $(n=414)$ & 64,0 & 24,6 & 11,4 \\
\hline Non $(n=1033)$ & 59,2 & 23,2 & 17,6 \\
\hline \multicolumn{4}{|l|}{ Syndicat } \\
\hline \multicolumn{4}{|c|}{ Tient compte de l'opinion des membres** } \\
\hline Oui $(\mathrm{n}=1159)$ & 67,5 & 23,9 & 8,6 \\
\hline Non $(n=285)$ & 43,2 & 24,9 & 31,9 \\
\hline \multicolumn{4}{|l|}{ Explique ses décisions** } \\
\hline Oui $(\mathrm{n}=1082)$ & 67,4 & 23,9 & 8,7 \\
\hline Non $(n=360)$ & 48,6 & 24,7 & 26,7 \\
\hline \multicolumn{4}{|c|}{ Position du syndicat vis-à-vis des changements } \\
\hline \multicolumn{4}{|l|}{ à l'organisation du travail } \\
\hline \multicolumn{4}{|c|}{ Exerce des pressions sur l'employeur } \\
\hline Oui $(\mathrm{n}=826)$ & 64,0 & 23,5 & 12,5 \\
\hline Non $(\mathrm{n}=585)$ & 60,7 & 25,1 & 14,2 \\
\hline \multicolumn{4}{|l|}{ S'oppose aux changements } \\
\hline Oui $(n=691)$ & 64,5 & 23,3 & 12,2 \\
\hline Non $(n=704)$ & 61,2 & 24,9 & 13,9 \\
\hline \multicolumn{4}{|c|}{ Travaille conjointement avec l'employeur } \\
\hline Oui $(\mathrm{n}=907)$ & 61,3 & 24,7 & 14,0 \\
\hline Non $(n=487)$ & 65,7 & 22,6 & 11,7 \\
\hline
\end{tabular}

$* \mathrm{p}<, 05 ; * * \mathrm{p}<, 005$

trois catégories. La première renvoie aux caractéristiques sociodémographiques des membres. Les femmes dans le secteur public sont 2,13 fois plus portées à considérer le syndicat peu essentiel que les hommes dans le secteur des biens. Cette distanciation des femmes du secteur public ne peut être attribuée exclusivement à leur localisation sectorielle ou aux pressions sur les conditions de travail. En effet, les hommes dans le secteur public ne se démarquent pas par rapport à ceux du secteur des biens, et aucune des variables privilégiées pour déterminer les pressions sur les conditions de travail n'est associée à l'appréciation du caractère essentiel du syndicalisme.

La deuxième catégorie de variables caractérise le comportement de l'employeur. D'un côté, l'hostilité de l'employeur accentue l'affaiblissement du lien syndicat-salarié : si l'employeur cherche à créer un climat d'intimidation $(1,54)$ ou se comporte en adversaire vis-à-vis du syndicat $(1,49)$, les probabilités sont plus fortes que le syndicat soit considéré peu essentiel. D'un autre côté, un employeur qui cherche à contourner la convention collective provoque l'effet inverse : dans un tel contexte, les membres sont moins portés à juger le syndicat peu essentiel. Ces résultats appuient 
TABLEAU 3

Estimations de la probabilité de croire au caractère essentiel du syndicalisme

\begin{tabular}{|c|c|c|c|}
\hline \multirow[b]{2}{*}{ Variables } & \multicolumn{3}{|c|}{ Estimations multiplicatives } \\
\hline & $\begin{array}{l}\text { Peu essentiel } \\
\text { contre essentiel }\end{array}$ & $\begin{array}{c}\text { Peu contre plutôt } \\
\text { essentiel }\end{array}$ & $\begin{array}{c}\text { Plutôt essentiel contre } \\
\text { essentiel }\end{array}$ \\
\hline \multicolumn{4}{|l|}{ Âge } \\
\hline$<30$ & 1,54 & 1,13 & 1,38 \\
\hline $30-39$ & 1,00 & 1,00 & 1,00 \\
\hline $40-49$ & 1,04 & 1,31 & 0,78 \\
\hline 50 et plus & 0,74 & 1,15 & 0,76 \\
\hline \multicolumn{4}{|l|}{ Scolarité } \\
\hline Secondaire et moins & 0,72 & 1,20 & 0,76 \\
\hline CEGEP & 1,00 & 1,00 & 1,00 \\
\hline Université & 0,68 & 1,03 & 0,72 \\
\hline \multicolumn{4}{|l|}{ Statut } \\
\hline Régulier temps complet & 1,00 & 1,00 & 1,00 \\
\hline Autre & 1,39 & $1,54 *$ & 1,03 \\
\hline \multicolumn{4}{|l|}{ Sexe/Secteur } \\
\hline Hommes fabrication & 1,00 & 1,00 & 1,00 \\
\hline Femmes services privés & 1,64 & 1,26 & $1,86^{*}$ \\
\hline Hommes services privés & 0,72 & 0,52 & $1,79^{*}$ \\
\hline Femmes services publics & $2,13^{*}$ & 1,08 & $1,81^{* *}$ \\
\hline Hommes services publics & 1,20 & 0,74 & $1,88^{*}$ \\
\hline \multicolumn{4}{|l|}{ Taille du syndicat } \\
\hline Petit $(<50)$ & 0,88 & 0,92 & 0,88 \\
\hline Moyen (50-249) & 1,00 & 1,00 & 1,00 \\
\hline Grand $(\geq 250)$ & 0,78 & $0,58^{*}$ & $1,30^{*}$ \\
\hline \multicolumn{4}{|l|}{ Conditions de travail } \\
\hline Satisfaction & 1,00 & 1,00 & 1,00 \\
\hline Insatisfaction & 1,03 & 1,03 & 0,96 \\
\hline Sécurité & 1,00 & 1,00 & 1,00 \\
\hline Insécurité & 0,86 & 0,99 & 1,02 \\
\hline Charge de travail stable & 1,00 & 1,00 & 1,00 \\
\hline Intensification de la charge & 0,83 & 0,98 & 0,83 \\
\hline \multicolumn{4}{|l|}{ Employeur } \\
\hline Ne se comporte pas en adversaire & 1,00 & 1,00 & 1,00 \\
\hline Se comporte en adversaire & $1,46^{*}$ & $1,49^{*}$ & 0,86 \\
\hline Ne contourne pas la convention collective & 1,00 & 1,00 & 1,00 \\
\hline Contourne la convention collective & $0,61^{*}$ & $0,67 *$ & 1,00 \\
\hline Ne crée pas un climat de peur & 1,00 & 1,00 & 1,00 \\
\hline Crée un climat de peur & $1,57^{*}$ & $1,54^{*}$ & 0,87 \\
\hline \multicolumn{4}{|l|}{ Syndicat } \\
\hline Tient compte de l'opinion des membres & 1,00 & 1,00 & 1,00 \\
\hline Ne tient pas compte de l'opinion des membres & $4,08 * *$ & $2,99 * *$ & 1,23 \\
\hline Explique ses décisions & 1,00 & 1,00 & 1,00 \\
\hline N'explique pas ses décisions & $2,48 * *$ & $1,79^{*}$ & 1,35 \\
\hline \multicolumn{4}{|c|}{ Position du syndicat vis-à-vis la réorganisation du travail } \\
\hline Exerce des pressions sur l'employeur & 0,75 & 1,02 & $0,71^{*}$ \\
\hline N'exerce pas de pressions sur l'employeur & 1,00 & 1,00 & 1,00 \\
\hline S'oppose aux changements & 1,01 & 0,97 & 0,94 \\
\hline Ne s'oppose aux changements & 1,00 & 1,00 & 1,00 \\
\hline Travaille conjointement avec l'employeur & $1,55^{*}$ & 1,09 & $1,30^{*}$ \\
\hline $\mathrm{Ne}$ travaille pas conjointement avec l'employeur & 1,00 & 1,00 & 1,00 \\
\hline $\mathrm{N}$ & 1032 & 499 & 1177 \\
\hline Pseudo $\mathrm{R}^{2}$ & 0,23 & 0,19 & 0,05 \\
\hline
\end{tabular}

$* \mathrm{p}<, 05 ; * * \mathrm{p}<, 005$ 
une hypothèse forte dans la littérature voulant que le comportement de l'employeur peut infléchir dans un sens ou dans l'autre l'opinion des salariés sur le syndicat (Gallie, 1996).

La troisième catégorie de variables se rapporte au comportement du syndicat. Si le syndicat ne tient pas compte de l'opinion des membres $(4,08)$, n'explique pas clairement ses décisions $(2,48)$ et travaille conjointement avec l'employeur pour résoudre les problèmes de réorganisation du travail $(1,55)$, les probabilités augmentent d'autant que le syndicat soit jugé peu essentiel. Trois remarques s'imposent ici. Premièrement, la représentation que les membres se font du fonctionnement démocratique du syndicat structure leur évaluation du caractère essentiel du syndicalisme. Ce lien peut paraître tautologique. Il peut sembler évident que les membres qui considèrent le syndicat peu essentiel ont davantage tendance à déprécier le fonctionnement démocratique du syndicat, notamment pour justifier leur position. Une telle interprétation ne résiste pas à l'analyse, car le lien entre l'appréciation du fonctionnement démocratique du syndicat et le caractère essentiel du syndicalisme est loin d'être univoque. Par exemple, une forte proportion de membres qui considèrent le syndicat comme peu essentiel estiment néanmoins démocratique le fonctionnement de leur syndicat. L'inverse est également vrai. En ce sens, l'appréciation du fonctionnement démocratique ne détermine pas complètement le jugement que portent les membres sur le caractère essentiel du syndicalisme. Deuxièmement, dans la mesure où la position du syndicat à l'égard des changements à l'organisation du travail n'influence pas directement l'évaluation du syndicalisme, on peut tenter de la relier aux autres variables syndicales, à savoir la perception du fonctionnement démocratique du syndicat. Dans cette perspective, si une position d'appui syndical influence l'évaluation du caractère essentiel du syndicalisme, ce ne serait qu'à travers le prisme du fonctionnement du syndicat local. Dans un contexte où le syndicat travaille conjointement avec l'employeur mais ne tient pas compte de l'opinion des membres et n'explique pas clairement ses décisions, il y a 5,43 plus de chances que le syndicat apparaisse peu essentiel. Ainsi, dans la mesure où les responsables syndicaux ne font pas preuve de transparence dans leur travail conjoint avec l'employeur, l'implication du syndicat peut réduire sa capacité à mobiliser les membres, voire atténuer leur attachement à l'institution syndicale. Troisièmement, le comportement du syndicat semble avoir plus d'influence sur l'évaluation du caractère essentiel du syndicalisme que le comportement de l'employeur. Ces résultats suggèrent que les syndicats disposent d'une marge de manœuvre pour façonner cette première dimension de l'identité syndicale, car elle est loin d'être uniquement conditionnée par des facteurs extragènes aux syndicats. Au contraire, elle semble tributaire de l'action du syndicat, notamment son fonctionnement démocratique.

La comparaison entre les membres jugeant le syndicalisme peu et plutôt essentiel confirme ces tendances (colonne 2). D'abord, les variables caractérisant le fonctionnement démocratique du syndicat sont de nouveau fortement associées à l'évaluation du caractère essentiel du syndicalisme. Il en va de même des variables renvoyant aux comportements de l'employeur. Dans un contexte où l'employeur se comporte en adversaire et tente de créer un climat d'intimidation, les probabilités sont plus fortes que le membre considère le syndicat comme peu essentiel. Bref, le contexte dans lequel s'insère le membre a un effet structurant majeur sur son appréciation du caractère essentiel du syndicalisme. Ensuite, ni la localisation sectorielle, ni la scolarité, ni l'âge ne semblent atténuer ou accentuer l'attachement des membres envers le syndicalisme. En revanche, la taille du syndicat et le statut d'emploi permettent de départager les membres estimant que le syndicat est plutôt ou peu essentiel. Dans les grands syndicats, en comparaison de ceux de taille moyenne, les membres ont moins tendance à considérer le syndicat comme peu essentiel. Si la taille semble atténuer le détachement des membres à l'égard du syndicalisme, il en va autrement du statut d'emploi. Les membres occupant un emploi précaire ont 1,54 fois plus de chances que ceux occupant un emploi régulier de juger le syndicat peu essentiel.

À la dernière colonne du tableau 4 , on retrouve les variables permettant de distinguer les membres considérant le syndicat comme essentiel de ceux qui le jugent plutôt essentiel. Il apparaît clairement que l'appréciation de ces membres n'est pas liée au fonctionnement démocratique du syndicat. Le comportement de l'employeur n'infléchit pas non plus l'évaluation des membres du 
caractère essentiel du syndicat, contrairement à ce qu'on a pu observer dans le cas des membres considérant le syndicat comme peu essentiel. Bref, les variables exerçant le plus d'influence sur les membres jugeant le syndicat peu essentiel ne permettent pas de départager ceux qui considèrent le syndicalisme comme plutôt essentiel et essentiel.

Parmi les variables caractérisant les acteurs, seule la position du syndicat à l'égard de la réorganisation du travail permet de distinguer les membres considérant le syndicalisme comme essentiel de ceux qui le jugent plutôt essentiel. Deux tendances semblent se dessiner : d'une part un syndicat qui exerce des pressions sur l'employeur pour modifier l'organisation du travail accentue la propension à juger que le syndicat est essentiel ; d'autre part, un syndicat qui travaille conjointement avec l'employeur pour résoudre les problèmes de réorganisation contrecarre cette même tendance. On serait donc porté à soutenir qu'un syndicat pro-actif dans le domaine de la réorganisation du travail renforce l'attachement des membres à l'égard du syndicalisme. Outre la position du syndicat, la localisation sectorielle et la taille de l'unité permettent de départager les membres qui croient que le syndicat est essentiel et plutôt essentiel. Quelle que soit la base de comparaison, les hommes dans le secteur des biens sont plus enclins à croire que le syndicat est essentiel. La même tendance s'observe chez les membres des grandes unités.

De l'ensemble de ces données, il se dégage quatre constats. Premièrement, l'affaiblissement du rapport salarié-syndicat, du moins en ce qui concerne le caractère essentiel du syndicat, en plus d'être moins marqué que ne le laissent présager certaines thèses, ne suit pas une trajectoire sociodémographique univoque. Ni la scolarité, ni l'âge du répondant n'affectent dans un sens ou dans l'autre l'opinion des membres relativement au caractère essentiel du syndicat. Toutefois, la précarité de l'emploi semble se traduire par une plus forte distanciation à l'égard du syndicat, en particulier lorsque les membres considérant le syndicat comme peu essentiel sont comparés à ceux le jugeant plutôt essentiel. Il en va de même pour la taille du syndicat. Quant à la localisation sectorielle et la composition sexuelle de la main-d'œuvre, les résultats s'avèrent moins variables. Les femmes dans les services publics ont plus tendance à considérer le syndicat comme peu essentiel, en comparaison des hommes dans le secteur des biens. Sans atténuer la portée de ce résultat, il ressort des données que les hommes dans le secteur des biens sont plus enclins que toutes les autres catégories à juger le syndicat essentiel.

Deuxièmement, les fluctuations dans les conditions de travail et le degré de satisfaction des membres à l'égard de leurs conditions de travail ne sont pas associées à l'évaluation du caractère essentiel du syndicalisme. En d'autres termes, si le syndicat est jugé peu essentiel, ce n'est pas parce qu'on est insatisfait de ses conditions de travail. On pourra en conclure que l'attachement au syndicat ne repose pas strictement sur une logique instrumentale du rôle du syndicat dans l'amélioration des conditions de travail. Il ne s'agit pas ici de soutenir que les membres ne procèdent pas à une telle évaluation. Il s'agit plutôt de souligner que ce jugement tient à l'évaluation du comportement des acteurs dans le milieu du travail.

Le troisième constat qui ressort de cette analyse concerne l'évaluation du comportement de l'employeur. Dans un contexte où l'employeur se comporte en adversaire et crée un climat d'intimidation, les membres sont davantage enclins à considérer le syndicalisme comme peu essentiel, mais contourner la convention collective peut atténuer la distanciation à l'égard de l'institution syndicale. Ces résultats montrent bien que l'appui à l'institution syndicale est une source de tensions et un enjeu de pouvoir dans les milieux de travail. Autrement dit, l'employeur n'est pas un acteur neutre et désintéressé dans l'attachement des membres à leur syndicat. Ses actions peuvent accentuer ou atténuer leur attachement.

Toutefois, le syndicat n'est pas complètement démuni face à un employeur hostile. En fait, il dispose d'une marge de manœuvre relative : il peut atténuer la portée des actions de l'employeur et le détachement des membres envers le syndicat. Le fonctionnement démocratique du syndicat apparaît à la fois comme une exigence et comme une condition expresse de l'adhésion des membres au syndicat. Tel est le quatrième constat qui ressort de cette analyse. La probabilité qu'un membre considère le syndicat comme peu essentiel est nettement plus élevée lorsqu'il juge que le syndicat ne 
tient pas compte de l'opinion des membres ou encore n'explique pas clairement ses décisions. Si, dans son fonctionnement interne, le syndicat s'isole de ses membres, les risques sont d'autant plus élevés que les membres jugent le syndicat peu essentiel. Cette tendance apparaît d'autant plus forte dans un contexte où le syndicat travaille conjointement avec l'employeur pour résoudre les problèmes de réorganisation du travail. On retiendra donc ici que le fonctionnement démocratique du syndicat constitue la condition première de l'attachement des membres au syndicat, condition qui se révèle d'autant plus importante dans un contexte où le syndicat s'engage dans des démarches de régulation conjointe du changement.

\section{LES MODALITÉS D'ACTION COLLECTIVE}

Le syndicalisme industriel se caractérise, entre autres, par un ensemble de valeurs et de représentations concernant les pratiques et les actions syndicales. La seconde dimension retenue pour cerner l'identité syndicale cherche à circonscrire les contours de l'adhésion des membres aux modalités d'action collective traditionnelles. Trois questions cherchaient à cerner ces valeurs. La première concerne le principe d'aire de représentation des syndicats : « Les syndicats doivent défendre tous les travailleurs dans la société, même les travailleurs non syndiqués. » À cette question, 37,1\% des répondants ont indiqué qu'ils étaient tout à fait d'accord, 32,3\% plutôt d'accord, 19,1\% plutôt en désaccord et $11,5 \%$ tout à fait en désaccord. La deuxième a trait à l'utilité de la grève comme moyen de pression : «La grève demeure toujours un moyen indispensable pour défendre les droits des travailleurs. » Les réponses à cette question montrent une adhésion moins forte, ce qui n'est pas étranger au fait qu'une forte proportion des membres travaillent dans le secteur public. De fait, $17,3 \%$ des répondants étaient tout à fait d'accord avec cet énoncé, 22,8 \% plutôt d'accord, 34,6 \% plutôt en désaccord et $25,3 \%$ tout à fait en désaccord. La dernière question tente de délimiter, par la négative, le principe de solidarité entre les salariés lors d'un conflit de travail : «Un syndiqué qui ne souhaite pas faire la grève devrait avoir le droit de traverser les lignes de piquetage pour travailler. » À cette question, 19,5\% ont exprimé un accord sans réserve, 12,9\% un accord mitigé, $20,3 \%$ ont indiqué qu'ils étaient plutôt en désaccord et 47,3 \% tout à fait en désaccord.

De manière à obtenir un portrait synoptique, les réponses à ces trois questions ont été combinées en utilisant l'analyse en grappes ${ }^{14}$. Cette procédure a permis de dégager trois profils d'adhésion. Le premier profil regroupe 488 membres $(35,8 \%)$ qui appuient l'ensemble des principes traditionnels de l'action collective. En plus de juger que le syndicat doit défendre tous les travailleurs dans la société, même les non-syndiqués, ils estiment que la grève est indispensable et qu'un salarié ne devrait pas avoir le droit de traverser les lignes de piquetage. Les deux autres profils se démarquent l'un de l'autre tant en nature qu'en intensité. Ainsi, le deuxième profil $(n=453)$ réunit des membres qui ne considèrent pas que la grève soit indispensable ni que les syndicats devraient protéger les travailleurs non-syndiqués. Par contre, ils jugent qu'un employé ne devrait pas avoir le droit de traverser la ligne de piquetage lors d'un conflit. Bref, leur adhésion, qui peut être qualifiée de modérée, repose sur un principe de solidarité limitée aux salariés en grève. Les membres classés dans le troisième profil $(n=453)$ ont plutôt tendance à juger que les syndicats doivent défendre les intérêts des non-syndiqués, mais ils estiment par ailleurs que la grève n'est pas indispensable et qu'un salarié devrait avoir le droit de traverser une ligne de piquetage. On passe ainsi d'un groupe dont l'adhésion se confine à la protection des droits collectifs des syndiqués en grève à un groupe qui valorise les droits individuels dans le cadre d'une représentation syndicale plus large. Cette tendance à faire primer les droits individuels nous conduit à qualifier de faible l'adhésion de ces membres aux modalités d'action collective traditionnelles.

14. L'analyse en grappes (cluster analysis) est une procédure statistique qui permet de constituer empiriquement des groupes relativement homogènes. L'objectif est d'établir des groupes de façon telle que la variance intergroupe soit maximale et la variance intragroupe minimale. Contrairement à l'analyse factorielle, qui est généralement utilisée pour effectuer des regroupements de variables, l'analyse en grappes permet de regrouper des unités d'observation sur la base de différentes variables caractérisant les unités d'observation. 
Comme on peut l'observer à la lecture du tableau 4, l'adhésion des membres aux modalités d'action collective varie de façon significative selon le sexe, l'âge, la scolarité, le statut d'emploi et la localisation sectorielle. Ainsi, les hommes sont davantage enclins à adhérer aux modalités d'action collective que les femmes : 45,5 \% d'entre eux y adhèrent fortement, pourcentage qui chute à $25,4 \%$ chez les femmes. Les jeunes ont également tendance à se démarquer par rapport aux autres cohortes d'âges : seulement $21,2 \%$ des membres de moins de trente ans souscrivent fortement à ces modalités d'action, la majorité d'entre eux $(50,6 \%)$ exprimant plutôt une faible adhésion. Le statut d'emploi semble produire un clivage identique à celui observé pour l'âge. Les membres ayant un emploi régulier à temps complet sont beaucoup plus enclins à appuyer les modalités d'action collective que les membres ayant un emploi non standard, dont l'adhésion demeure fragile (47,7 \%). Les salariés ayant une formation collégiale sont également moins portés à appuyer de façon inconditionnelle les modalités d'action collective $(26,8 \%)$ et davantage enclins à les rejeter $(38,8 \%)$. Il en va de même pour les membres dans le secteur public : près de $40 \%$ d'entre eux expriment une faible adhésion aux modalités d'action, alors que dans le secteur des biens et dans les services privés, ce pourcentage chute respectivement à $20,3 \%$ et $19,0 \%$.

TABLEAU 4

Adhésion des salariés aux modalités d'action collective traditionnelles selon les sources de différenciation sociale

\begin{tabular}{|c|c|c|c|}
\hline & Forte adhésion & Adhésion moyenne & Faible adhésion \\
\hline \multicolumn{4}{|l|}{$\operatorname{Sexe}^{* *}$} \\
\hline Homme $(n=640)$ & 45,5 & 31,6 & 23,0 \\
\hline Femme $(\mathrm{n}=776)$ & 25,4 & 35,2 & 39,4 \\
\hline \multicolumn{4}{|l|}{$\hat{\mathbf{A}} \mathbf{e}^{* *}$} \\
\hline Moins de 30 ans $(n=170)$ & 21,2 & 28,2 & 50,6 \\
\hline Entre 30 et 39 ans $(n=502)$ & 33,5 & 37,3 & 29,3 \\
\hline Entre 40 et 49 ans $(n=494)$ & 37,2 & 33,2 & 29,6 \\
\hline Plus de 50 ans $(n=25)$ & 40,0 & 30,4 & 29,6 \\
\hline \multicolumn{4}{|l|}{ Scolarité** } \\
\hline Secondaire ou moins $(n=747)$ & 39,2 & 32,1 & 28,6 \\
\hline Formation collégiale $(n=400)$ & 26,8 & 34,5 & 38,8 \\
\hline Formation universitaire $(n=268)$ & 32,8 & 35,8 & 31,3 \\
\hline \multicolumn{4}{|l|}{ Statut d'emploi*** } \\
\hline Temps complet régulier $(\mathrm{n}=973)$ & 38,3 & 36,7 & 25,0 \\
\hline Autres $(n=442)$ & 26,0 & 26,7 & 47,3 \\
\hline \multicolumn{4}{|l|}{ Secteur d'activité*** } \\
\hline Biens privés $(\mathrm{n}=310)$ & 46,5 & 34,5 & 19,0 \\
\hline Services privés $(\mathrm{n}=222)$ & 41,9 & 37,8 & 20,3 \\
\hline Services publics $(\mathrm{n}=884)$ & 28,4 & 32,1 & 39,5 \\
\hline \multicolumn{4}{|l|}{ Taille du syndicat } \\
\hline Moins de $50(n=207)$ & 35,7 & 39,6 & 24,6 \\
\hline Entre 50 et $249(\mathrm{n}=567)$ & 33,3 & 33,2 & 33,5 \\
\hline 250 et plus $(n=642)$ & 33,0 & 34,0 & 32,0 \\
\hline
\end{tabular}

$* \mathrm{p}<, 05 ; * * \mathrm{p}<, 005$

L'adhésion des membres aux modalités d'action fluctue aussi en fonction des caractéristiques du contexte. Comme on peut l'observer à la lecture du tableau 5, leur adhésion semble particulièrement sensible aux comportements de l'employeur et du syndicat et à la position de ce dernier en matière de réorganisation du travail. En revanche, elle ne varie pas selon l'évaluation qu'ils font de leurs conditions de travail.

Parmi les variables retenues pour caractériser le comportement de l'employeur, une seule est significativement associée à l'adhésion des membres aux modalités d'action collective. Dans un contexte où l'employeur tente de créer un climat d'intimidation, seulement 29,5\% souscrivent à ces valeurs, alors que ce pourcentage atteint $36,4 \%$ dans les milieux où l'employeur ne cherche pas à 
TABLEAU 5

Adhésion des salariés aux modalités d'action collective selon les variables caractérisant leur contexte matériel de travail

\begin{tabular}{|c|c|c|c|}
\hline & $\begin{array}{c}\text { Forte } \\
\text { adhésion }\end{array}$ & $\begin{array}{l}\text { Moyenne } \\
\text { adhésion }\end{array}$ & $\begin{array}{c}\text { Faible } \\
\text { adhésion }\end{array}$ \\
\hline \multicolumn{4}{|l|}{ Conditions de travail } \\
\hline \multicolumn{4}{|l|}{ Satisfaction au travail } \\
\hline Élevée (n = 735) & 33,1 & 32,4 & 34,6 \\
\hline Faible $(\mathrm{n}=681)$ & 36,0 & 34,8 & 29,2 \\
\hline \multicolumn{4}{|l|}{ Sécurité d'emploi } \\
\hline Maintenue $(\mathrm{n}=1065)$ & 35,6 & 33,5 & 30,9 \\
\hline Diminuée $(\mathrm{n}=345)$ & 31,6 & 33,6 & 34,8 \\
\hline \multicolumn{4}{|l|}{ Charge de travail } \\
\hline Maintenue $(\mathrm{n}=546)$ & 35,2 & 31,1 & 33,7 \\
\hline Augmentée $(\mathrm{n}=862)$ & 33,9 & 35,3 & 30,9 \\
\hline \multicolumn{4}{|l|}{ Employeur } \\
\hline \multicolumn{4}{|c|}{ Se comporte en adversaire } \\
\hline Non $(n=780)$ & 34,9 & 32,1 & 33,1 \\
\hline Oui $(\mathrm{n}=636)$ & 34,0 & 35,4 & 30,7 \\
\hline \multicolumn{4}{|c|}{ Cherche à contourner la convention collective } \\
\hline Non $(n=654)$ & 33,5 & 32,4 & 34,1 \\
\hline Oui $(\mathrm{n}=752)$ & 35,0 & 34,7 & 30,3 \\
\hline \multicolumn{4}{|c|}{ Crée un climat d'intimidation* } \\
\hline Oui $(\mathrm{n}=403)$ & 29,5 & 35,5 & 35,0 \\
\hline Non $(n=1013)$ & 36,4 & 32,8 & 30,8 \\
\hline \multicolumn{4}{|l|}{ Syndicat } \\
\hline \multicolumn{4}{|c|}{ Tient compte de l'opinion des membres** } \\
\hline Oui $(\mathrm{n}=1133)$ & 36,6 & 34,7 & 28,7 \\
\hline Non $(n=280)$ & 26,1 & 28,9 & 45,0 \\
\hline \multicolumn{4}{|l|}{ Explique ses décisions** } \\
\hline Oui $(\mathrm{n}=1055)$ & 37,1 & 33,7 & 29,2 \\
\hline Non $(n=356)$ & 26,7 & 33,1 & 40,2 \\
\hline \multicolumn{4}{|c|}{ Position du syndicat vis-à-vis des changements } \\
\hline \multicolumn{4}{|c|}{ à l'organisation du travail } \\
\hline \multicolumn{4}{|c|}{ Exerce des pressions sur l'employeur } \\
\hline Oui $(\mathrm{n}=808)$ & 34,2 & 31,7 & 34,2 \\
\hline Non $(n=575)$ & 35,0 & 36,2 & 28,9 \\
\hline \multicolumn{4}{|l|}{ S'oppose aux changements } \\
\hline Oui $(\mathrm{n}=678)$ & 34,5 & 34,4 & 31,1 \\
\hline Non $(n=687)$ & 32,9 & 34,8 & 32,3 \\
\hline \multicolumn{4}{|c|}{ Travaille conjointement avec l'employeur* } \\
\hline Oui $(\mathrm{n}=889)$ & 35,0 & 32,1 & 33,0 \\
\hline Non $(n=475)$ & 31,2 & 39,8 & 29,1 \\
\hline
\end{tabular}

$* \mathrm{p}<, 05 ; * * \mathrm{p}<, 005$

créer un climat d'intimidation. De nouveau, les variables touchant le fonctionnement démocratique du syndicat sont liées à l'opinion des membres. Quand un membre considère que le syndicat tient compte de l'opinion des membres ou qu'il explique clairement ses décisions, il est davantage enclin à adhérer aux modalités d'action collective. En revanche, lorsqu'il exprime l'opinion opposée, il a moins tendance à adhérer à ces valeurs. Les écarts sont ici assez prononcés. Par exemple, parmi les membres jugeant que le syndicat ne tient pas compte de l'opinion de ses membres, 45,0\% manifestent une faible adhésion aux modalités d'action, pourcentage qui chute à $28,7 \%$ dans le cas des membres estimant que le syndicat tient compte de l'opinion des membres. Le lien entre la position du syndicat en matière d'organisation du travail et l'opinion des membres est plus équivoque. Dans un contexte où le syndicat ne travaille pas conjointement avec l'employeur, les membres ont plutôt tendance à adhérer modérément aux modalités d'action collective ; dans un contexte où il travaille conjointement avec l'employeur, les membres ont soit tendance à adhérer fortement aux modalités d'action collective, soit tendance à y souscrire faiblement. 
De manière à obtenir un portrait global des facteurs qui influencent le degré d'adhésion des membres aux modalités d'action collective, l'ensemble des variables a, de nouveau, été pris en considération concurremment. Il s'agissait de la même façon d'opposer chacune des paires afin d'identifier les variables les plus susceptibles d'infléchir cette dimension de l'identité syndicale. Des données exposées au tableau 6, il se dégage trois constats généraux. D'abord, les variables caractérisant les sources potentielles de différenciation sociale structurent fortement l'adhésion des membres aux modalités d'action collective. Ainsi, même lorsque les variables contextuelles sont introduites, le statut d'emploi, la scolarité, l'âge des répondants et la variable combinant la localisation sectorielle et la composition sexuelle de la main-d'œuvre sont significativement associées, à une étape ou l'autre de l'analyse, à l'adhésion des membres aux modalités d'action. Ensuite, si le jugement que portent les membres sur leurs conditions de travail n'est pas directement lié à leur adhésion aux modalités d'action collective, il l'est lorsque l'ensemble des variables est pris en considération simultanément. Inversement, l'effet des variables désignant le comportement de l'employeur s'estompe lorsque toutes les variables sont introduites simultanément. Enfin, le fonctionnement démocratique du syndicat et, dans une moindre mesure, sa position vis-à-vis des changements à l'organisation du travail concourent également à départager les membres selon leur degré d'adhésion aux modalités d'action collective.

Des données exposées au tableau 6, la remarque la plus évidente est que les femmes dans le secteur public, comparativement aux hommes dans le secteur des biens, ont 5,5 fois plus de chances d'adhérer faiblement aux modalités d'action collective (tableau 6, première colonne). On observe la même tendance mais de façon moins marquée chez les hommes du secteur public $(2,0)$, les jeunes $(2,7)$, les membres occupant un emploi précaire $(1,7)$ et ceux avec une formation collégiale $(1,79)$. En conjuguant ces caractéristiques, les probabilités que les femmes du secteur public de moins de trente ans ayant un emploi précaire souscrivent faiblement aux modalités d'action collective sont 7,11 fois plus élevées. Dans le cas des hommes ayant les mêmes caractéristiques, cette probabilité se situe à 3,61. Force est donc de reconnaître que l'affaiblissement des valeurs collectives traditionnelles est plus prononcé dans le secteur public, en particulier parmi les femmes de moins de trente ans ayant un statut précaire. Cela dit, l'évaluation que font les membres de leurs conditions de travail semble atténuer cette brisure. Les membres insatisfaits de leurs conditions de travail ont plus tendance à adhérer fortement aux modalités d'action collective. Il en va de même pour les membres qui jugent que leur charge de travail a augmenté au cours des trois dernières années. Les pressions sur les conditions de travail semblent ainsi accentuer l'adhésion aux modalités d'action traditionnelles.

De nouveau, l'opinion des membres quant au fonctionnement démocratique du syndicat a une incidence non négligeable sur le rapport salarié-syndicat. Les probabilités sont plus grandes qu'ils manifestent une adhésion faible plutôt que forte aux modalités d'action collective lorsqu'ils estiment que le syndicat ne tient pas compte de l'opinion des membres $(1,8)$ ou n'explique pas clairement ses décisions $(1,7)$. En couplant ces deux indicateurs, les probabilités qu'un membre souscrive faiblement à ces modalités atteignent 2,36. Lorsqu' un membre porte un jugement défavorable sur le fonctionnement démocratique du syndicat et qu'en plus le syndicat travaille conjointement avec l'employeur en matière de réorganisation du travail, les probabilités qu'il adhère faiblement aux modalités d'action grimpent à 2,79. Ces résultats renforcent l'hypothèse que l'implication du syndicat dans les projets de réorganisation doit s'accompagner de plus de transparence et d'ouverture vis-à-vis des membres du syndicat.

Si le fonctionnement démocratique du syndicat et sa position en matière d'organisation ont une incidence importante sur l'adhésion des membres aux modalités d'action, il n'en demeure pas moins que les sources de différenciation sociale semblent avoir un effet plus prononcé. Les femmes et les hommes dans le secteur public, les jeunes, les membres occupant un emploi précaire et ceux avec une formation collégiale ou universitaire ont plus tendance à adhérer faiblement aux modalités d'action collective. 
TABLEAU 6

Estimations de la probabilité d'adhésion aux modalités d'action collective

\begin{tabular}{|c|c|c|c|}
\hline \multirow[b]{2}{*}{ Variables } & \multicolumn{3}{|c|}{ Estimations multiplicatives } \\
\hline & $\begin{array}{l}\text { Faible contre } \\
\text { forte } \\
\text { adhésion }\end{array}$ & $\begin{array}{l}\text { Faible contre } \\
\text { moyenne } \\
\text { adhésion }\end{array}$ & $\begin{array}{l}\text { Moyenne } \\
\text { contre forte } \\
\text { adhésion }\end{array}$ \\
\hline \multicolumn{4}{|l|}{ Ȧge } \\
\hline$<30$ & $2,76^{* *}$ & $2,04 * *$ & 1,02 \\
\hline $30-39$ & 1,00 & 1,00 & 1,00 \\
\hline $40-49$ & 0,90 & 1,11 & 0,71 \\
\hline 50 et plus & 0,87 & 1,12 & $0,64 *$ \\
\hline \multicolumn{4}{|l|}{ Scolarité } \\
\hline Secondaire et moins & $0,72^{*}$ & 0,95 & 0,75 \\
\hline CEGEP & 1,00 & 1,00 & 1,00 \\
\hline Université & $0,55^{*}$ & $0,67^{*}$ & 0,70 \\
\hline \multicolumn{4}{|l|}{ Statut } \\
\hline Régulier temps complet & 1,00 & 1,00 & 1,00 \\
\hline Autre & $1,77^{* *}$ & $2,24 * *$ & 0,79 \\
\hline \multicolumn{4}{|l|}{ Sexe/Secteur } \\
\hline Hommes fabrication & 1,00 & 1,00 & 1,00 \\
\hline Femmes services privés & 1,63 & 0,91 & 1,52 \\
\hline Hommes services privés & 0,80 & 1,13 & 0,67 \\
\hline Femmes services publics & $5,52 * *$ & $2,36^{* *}$ & $2,24 * *$ \\
\hline Hommes services publics & $2,02 * *$ & $1,86^{*}$ & 1,00 \\
\hline \multicolumn{4}{|l|}{ Taille du syndicat } \\
\hline Petit $(<50)$ & 0,73 & 0,83 & 0,92 \\
\hline Moyen (50-249) & 1,00 & 1,00 & 1,00 \\
\hline Grand $(\geq 250)$ & 0,89 & 0,86 & 0,93 \\
\hline \multicolumn{4}{|l|}{ Conditions de travail } \\
\hline Satisfaction & 1,00 & 1,00 & 1,00 \\
\hline Insatisfaction & $0,60 * *$ & $0,68^{*}$ & 0,86 \\
\hline Sécurité & 1,00 & 1,00 & 1,00 \\
\hline Insécurité & 1,14 & 1,05 & 1,03 \\
\hline Charge de travail stable & 1,00 & 1,00 & 1,00 \\
\hline Intensification de la charge & $0,70^{*}$ & $0,73^{*}$ & 1,08 \\
\hline \multicolumn{4}{|l|}{ Employeur } \\
\hline Ne se comporte pas en adversaire & 1,00 & 1,00 & 1,00 \\
\hline Se comporte en adversaire & 1,22 & 0,90 & 1,15 \\
\hline $\mathrm{Ne}$ contourne pas la convention collective & 1,00 & 1,00 & 1,00 \\
\hline Contourne la convention collective & 0,77 & 0,81 & 1,04 \\
\hline Ne crée pas un climat de peur & 1,00 & 1,00 & 1,00 \\
\hline Crée un climat de peur & 1,04 & 0,87 & 1,28 \\
\hline \multicolumn{4}{|l|}{ Syndicat } \\
\hline Tient compte de l'opinion des membres & 1,00 & 1,00 & 1,00 \\
\hline Ne tient pas compte de l'opinion des membres & $1,82 * *$ & $1,87 * *$ & 0,94 \\
\hline Explique ses décisions & 1,00 & 1,00 & 1,00 \\
\hline N'explique pas ses décisions & $1,71 * *$ & 1,30 & $1,45^{*}$ \\
\hline \multicolumn{4}{|c|}{ Position du syndicat vis-à-vis la réorganisation du travail } \\
\hline Exerce des pressions sur l'employeur & 0,97 & 1,20 & 0,79 \\
\hline N'exerce pas de pressions sur l'employeur & 1,00 & 1,00 & 1,00 \\
\hline S'oppose aux changements & 1,13 & 0,86 & $1,36^{*}$ \\
\hline Ne s'oppose aux changements & 1,00 & 1,00 & 1,00 \\
\hline Travaille conjointement avec l'employeur & $1,53 *$ & 1,06 & $1,57 * *$ \\
\hline Ne travaille pas conjointement avec l'employeur & 1,00 & 1,00 & 1,00 \\
\hline $\mathrm{N}$ & 874 & 869 & 909 \\
\hline Pseudo $\mathrm{R}^{2}$ & 0,27 & 0,16 & 0,08 \\
\hline
\end{tabular}

$* \mathrm{p}<, 05 ; * * \mathrm{p}<, 005$ 
L'analyse comparative des membres souscrivant modérément et faiblement aux modalités d'action produit sensiblement les mêmes résultats à deux exceptions près (colonne 2). D'abord, la position du syndicat en matière d'organisation du travail et l'évaluation des membres quant à la façon dont le syndicat explique ses décisions ne sont pas significativement associées à l'adhésion des membres aux modalités d'action. Ensuite, l'écart entre les probabilités relatives associées à chacune des variables est nettement moins prononcé que dans l'analyse précédente. Cette situation s'explique par le fait que la probabilité que les femmes dans les services publics adhèrent faiblement plutôt que modérément aux modalités d'action collective se situe à 2,3, alors que dans l'analyse précédente, elle atteignait 5,5. Inversement, la probabilité associée à un statut précaire passe de 1,7 , dans l'analyse précédente, à 2,2, lorsque la comparaison porte sur les membres adhérant moyennement et faiblement aux modalités d'action collective. Au-delà de ces différences, les résultats concordent, ce qui ajoute à la robustesse de l'analyse.

La situation se présente différemment lorsque nous comparons les membres souscrivant fortement et modérément aux modalités d'action collection, même si les femmes dans le secteur public se démarquent de nouveau. Elles ont en effet 2,2 fois plus de chances d'adhérer modérément plutôt que fortement aux modalités d'action collective. Par contre, ni la scolarité, ni le statut d'emploi ne permettent de départager l'opinion de ces membres. En ce qui concerne l'âge, ce ne sont pas tant les jeunes comme les membres dans la quarantaine et la cinquantaine qui se distinguent. Ils sont plus enclins à adhérer fortement aux modalités d'action collective que les membres dans la trentaine. Quant aux variables caractérisant le contexte d'action, trois variables sont liées à l'opinion des membres. La première renvoie au fonctionnement démocratique du syndicat : les membres jugeant que le syndicat n'explique pas clairement ses décisions ont 1,4 fois plus de chances d'adhérer modérément plutôt que fortement aux modalités d'action collective. Les deux autres variables se rapportent à la position du syndicat en matière d'organisation du travail : que le syndicat s'oppose aux changements ou qu'il travaille conjointement avec l'employeur, les probabilités sont plus fortes que le membre souscrive modérément aux modalités d'action. Ces résultats suggèrent que ce n'est pas tant la position du syndicat qui importe que sa capacité à légitimer cette position auprès de ses membres.

De l'ensemble de cette analyse sur l'adhésion des membres aux modalités d'action collective, on peut tirer deux conclusions provisoires. D'abord, les sources de différenciation sociale semblent avoir un certain impact sur l'adhésion des membres aux modalités d'action collective. Les femmes dans le secteur public, les jeunes, les salariés avec un emploi précaire et ceux avec une formation collégiale sont moins portés à adhérer aux modalités d'action collective. De fait, ces membres ne semblent pas se reconnaître dans cette forme de syndicalisme. Ils valorisent soit les droits individuels dans le cadre d'une représentation syndicale plus large, soit un principe de solidarité limitée. Ensuite, même si ces sources de différenciation sociale apparaissent déterminantes, il n'en reste pas moins que le contexte matériel influence la propension des membres à adhérer aux modalités d'action collective traditionnelles. Outre une détérioration des conditions de travail qui peut renforcer l'adhésion à ces valeurs, le syndicat peut également agir sur ce plan. Le défi consiste dès lors à imaginer des modes de fonctionnement qui favorisent l'inclusion plutôt que l'exclusion de ces catégories de salariés.

\section{DISCUSSION ET CONCLUSION}

Les sources de différenciation qui caractérisent la société contemporaine provoquent-elles un affaiblissement du lien syndical ? Avant de rassembler les éléments de réponse à cette question, rappelons que notre objectif ne visait pas à cerner l'ensemble des facteurs susceptibles d'infléchir les identités syndicales, ce qui aurait impliqué une analyse approfondie des facteurs de socialisation, notamment familiale. Il s'agissait d'évaluer le mérite de deux thèses, notamment la thèse de la différenciation sociale qui, à notre connaissance, n'a pas fait l'objet d'une vérification empirique approfondie. 
De l'ensemble de cette analyse, il se dégage quatre constats qui représentent autant de pistes de réflexion. Premier constat : l'affaiblissement du lien au syndicat, notamment quant à son caractère essentiel, est nettement moins prononcé que ne le laissent présager certaines thèses. Une minorité de salariés syndiqués composant notre échantillon ne croient pas que le syndicalisme est essentiel. Règle générale, on constate au contraire un appui massif. Toutefois, l'adhésion aux modalités d'action collective traditionnelles est beaucoup plus nuancée et partagée. Ces résultats confortent l'idée selon laquelle l'enjeu des transformations sociales en cours est moins une crise du syndicalisme qu'une crise d'une forme singulière de syndicalisme qu'il s'agit de redéfinir à la lumière de ces transformations (voir, en particulier, Hyman, 1994). Ce constat vaut au premier chef dans le secteur public.

Deuxième constat: les sources de différenciation sociale ne provoquent pas un affaiblissement du lien syndical en tant que tel, du moins en ce qui a trait à son caractère essentiel, mais elles infléchissent lourdement l'adhésion aux modalités d'action. Seule la localisation sectorielle et la composition sexuelle de la main-d'œuvre influent largement sur l'opinion qu'on a du caractère essentiel du syndicat, les femmes dans le secteur public étant davantage portées à considérer le syndicat comme peu essentiel. L'affaiblissement du lien syndical parmi le personnel féminin dans les services publics dépasse, comme nous l'avons déjà constaté, une simple évaluation négative du caractère instrumental du syndicat dans l'amélioration des conditions de travail. Il s'agit d'un affaiblissement qui mérite explication car il ne se manifeste pas de la même façon chez les hommes de ce même secteur ${ }^{15}$. Relève-t-il, par exemple, d'un sentiment d'exclusion des sphères décisionnelles du syndicat, du sentiment que le syndicat ne se soucie pas des intérêts des femmes dans ce secteur, ou encore que le syndicat ne peut promouvoir leurs intérêts ? Des recherches futures devraient étudier cette question centrale pour l'évolution du syndicalisme.

L'analyse des modalités d'action collective présente un portrait plus complexe et plus lourd de conséquences pour l'acteur syndical. L'ensemble des sources de différenciation sociale influence l'adhésion des membres aux modalités d'action collective. Outre le cas particulier des femmes dans les services publics, les hommes de ce même secteur, les jeunes, les membres ayant un statut d'emploi précaire et ceux ayant une formation collégiale adhèrent moins fortement aux modalités d'action collective. La présence simultanée de multiples sources de différenciation conduit à une diminution encore plus importante de l'adhésion à ces modalités d'action. Ces nouvelles catégories de salariés ont ainsi beaucoup moins tendance à épouser l'ensemble des valeurs collectives les plus associées au syndicalisme industriel. La fracture apparaît ici assez prononcée, en particulier en ce qui concerne le caractère indispensable de la grève. En effet, les membres qui adhèrent modérément ou faiblement aux modalités d'action ont en commun de croire que la grève n'est pas indispensable. Cette situation s'explique sans doute par le fait que l'encadrement juridique de la grève dans les services publics en limite la portée et que l'expérience récente des grèves dans ce secteur a laissé des séquelles. Elle découle aussi sans doute du fait que la grève, à tout le moins dans le secteur des services publics, affecte davantage le consommateur que l'employeur. Par contre, l'opinion des membres sur le caractère indispensable de la grève ne représente pas l'unique indicateur permettant de déterminer l'adhésion des membres aux modalités d'action collective.

Les femmes et les hommes dans le secteur public, les jeunes, les salariés occupant un emploi précaire et ceux ayant une formation collégiale se démarquent aussi quant à la délimitation de l'aire de représentation des syndicats et aux principes de solidarité lors d'un conflit. Rappelons que la majorité de ces membres se caractérisent par une adhésion modérée ou faible aux modalités d'action collective. Dans le premier cas, ils favorisent une représentation étroite plutôt qu'élargie de l'aire de représentation, alors que dans le second cas, ils privilégient les droits individuels au détriment des droits collectifs. Les valeurs collectives caractérisant le plus le syndicalisme industriel semblent ainsi avoir moins de prise parmi ces salariés.

15. Pour une analyse plus détaillée des transformations des milieux de travail et de l'évolution des identités syndicales dans le secteur de la santé et les services sociaux, voir Le Queux (1997). 
Troisième constat : le contexte immédiat de la représentation syndicale, en particulier les bases matérielles du rapport d'emploi et les comportements des acteurs, influence significativement le rapport des salariés à l'institution syndicale, que ce soit concernant le caractère essentiel du syndicat ou leur adhésion aux modalités d'action collective. Ce constat appelle plusieurs nuances. D'abord, ce n'est pas tant l'évaluation que les salariés font de leurs conditions de travail que les comportements de l'employeur et du syndicat qui ont un effet structurant sur le lien au syndicat. L'évaluation des conditions de travail par les membres ne joue que de façon circonscrite sur leur adhésion aux modalités d'action collective. Ensuite, le comportement de l'employeur n'influence pas l'adhésion des membres aux modalités d'action, mais plutôt leur évaluation du caractère essentiel du syndicalisme. Ce facteur joue davantage que les conditions de travail, mais il demeure ambivalent. Nos résultats n'appuient pas forcément la thèse de la cooptation, à savoir que l'employeur bienveillant diminue le besoin d'un syndicat dans le milieu du travail. Au contraire, c'est précisément l'employeur qui se comporte en adversaire et qui crée un climat de peur qui contribue à ce que les membres ont une propension moindre à juger le syndicalisme essentiel. Par contre, ne pas respecter la convention collective et vouloir saboter la régulation collective au travail peut stimuler l'adhésion au syndicalisme. Et ces résultats apparaissent d'autant plus probants que les informations colligées pour cerner le comportement de l'employeur proviennent à la fois des représentants syndicaux et des salariés. Enfin, le comportement du syndicat influence tant l'appréciation des membres du caractère essentiel du syndicat que leur adhésion aux modalités d'action collective. Dans un contexte où les membres ont le sentiment que le syndicat ne fonctionne pas démocratiquement, on observe un affaiblissement assez marqué du lien syndical. Sa position en matière d'organisation du travail est également associée à l'appréciation des membres, mais les résultats apparaissent ici plutôt équivoques. D'une part, l'appui du syndicat dans les démarches de changement augmente les probabilités que le membre considère le syndicat comme peu essentiel, a fortiori si le syndicat n'a pas un fonctionnement démocratique. Les exigences de transparence et de démocratie semblent ainsi plus fortes lorsque le syndicat s'engage sur la voie de la régulation conjointe du changement. D'autre part, l'adhésion des membres aux modalités d'action collective s'atténue lorsque le syndicat adopte soit une position d'appui, soit une position d'opposition, en particulier si le membre juge que le syndicat n'a pas un fonctionnement démocratique. Ces résultats peuvent paraître paradoxaux, mais ils illustrent que la légitimité de la position du syndicat est aussi déterminante que sa position effective en matière d'organisation du travail.

Quatrième constat : si on tente d'identifier la principale source d'affaiblissement du lien au syndicat, c'est la perception du fonctionnement démocratique du syndicat, et non pas les sources de différenciation sociale, qui se révèle la variable la plus structurante. Une perception positive du fonctionnement démocratique renforce le lien au syndicat : il s'agit du facteur le plus déterminant en ce qui concerne l'évaluation du caractère essentiel du syndicalisme. L'analyse des modalités d'action collective présente un portrait plus complexe, car les sources de différenciation sociale sont associées à un affaiblissement de l'adhésion aux valeurs du syndicalisme industriel. Ce résultat conforte dans une certaine mesure la thèse de la désagrégation sociale. On peut, à ce propos, évoquer l'effet structurant de nouvelles subjectivités sur le marché du travail qui remettent en cause les modalités d'action traditionnelles des organisations syndicales. Or, encore une fois, outre la dégradation des conditions de travail qui semble limiter la portée de l'affaiblissement lié aux sources de différenciation sociale, le seul facteur susceptible d'exercer un contrepoids est le fonctionnement démocratique du syndicat. Le renouveau des modalités d'action syndicales représente un défi plus important pour les syndicats. Les nouvelles catégories de salariés sont porteuses de nouvelles valeurs qui ne remettent pas en cause pour autant le caractère essentiel du syndicalisme mais qui doivent renouveler ses modes d'action. Autrement dit, il faut se demander comment on donnera la voix à ces nouvelles subjectivités dans les modes d'action syndicale. Néanmoins, les salariés syndiqués ne sont pas indifférents à la pratique syndicale. De façon générale, le lien au syndicat se structure autour de la légitimité des actions syndicales qui reposent elles-mêmes sur la mobilisation de la subjectivité des salariés, d'où l'importance d'examiner la démocratie syndicale dans ce nouveau contexte. 
L'implication de cette recherche pour notre compréhension de l'identité syndicale concerne avant tout la réception démocratique par les syndicats des nouvelles sources de différenciation sociale dans la société. Nous l'avons déjà constaté : la perception d'une pratique démocratique renforce, en définitive, l'adhésion aux valeurs collectives. Il ne s'agit pas ici, toutefois, de "culpabiliser" les responsables syndicaux, car c'est non seulement la perception de la démocratie mais, plus fondamentalement, la participation à la pratique démocratique qui permet à la fois l'adhésion aux valeurs collectives et leur renouvellement. Il incombe alors aux organisations syndicales de redéfinir les identités collectives pour mieux tenir compte des nouvelles sources de différenciation sociale. Les transformations sociales constitueraient, dans cette perspective, une occasion, mais aussi une obligation, de renouvellement des identités syndicales.

Ces résultats corroborent un certain discours sur les exigences du renouvellement syndical. Hyman (1994, p. 119) plaide, par exemple, pour une mise à jour des modes de participation et de décisions dans les organisations syndicales : « À mesure que les modalités d'organisation traditionnelles - le plus souvent hiérarchiques et bureaucratiques — se révèlent inefficaces, les systèmes de communication et de prise de décision doivent être repensés pour les adapter aux réalités sociales des années 1990 [...] À mesure que les méthodes traditionnelles de lutte semblent perdre de l'efficacité ou s'attaquer à une mauvaise cible, alors les syndicats, dans plusieurs cas, sont amenés à s'interroger sur la nature et les objectifs des actions qu'ils mènent [...] » (C'est nous qui traduisons). Heckscher (1988, p. 65), prône une approche semblable quand il se demande si les difficultés syndicales relèvent d'un manque de solidarité ou de l'incapacité des structures actuelles à répondre aux nouvelles formes de solidarité.

Les nouvelles sources de différenciation sociale interpelleraient ainsi l'acteur syndical dans sa fonction démocratique, non seulement sur sa base représentative, mais aussi en ce qui concerne ses modes de participation et la prise en compte de variations dans le rapport individuel à l'institution syndicale. À ce titre, il convient à nouveau de souligner que la chose collective n'est pas évidente en soi. Elle relève d'une discipline permettant l'alignement des intérêts individuels, ou du moins une régulation commune (Reynaud, 1989), sans faire abstraction des intérêts des acteurs concernés. Elle relève aussi foncièrement d'une socialisation, d'où l'importance de la participation : «La solidarité n'est pas une condition posée a priori, mais plutôt le résultat d'efforts répétés dans le but de développer des complicités et des alliances entre travailleurs malgré les différences qui les séparent. Et on ne peut y arriver que par une participation large aux activités et débats syndicaux. » (Leisink et coll., 1996, p. 21 ; c'est nous qui traduisons.)

Les sources de différenciation sociale à l'étude dans ce texte ne constituent pas une rupture par rapport au syndicalisme en tant qu'institution représentative des salariés syndiqués. Elles appellent, par contre, à un renouveau de ses modalités d'action. Ce renouveau doit s'inspirer d'un double mouvement : d'une part, l'élaboration de nouvelles valeurs et de nouveaux modes d'action reflétant les nouvelles identités sur le marché du travail doit partir de l'implication de ces groupes de salariés dans les débats syndicaux; d'autre part, la transparence démocratique dans ces débats et le fait même d'y participer renforceront l'adhésion aux nouvelles valeurs collectives en émergence.

\section{Christian LÉVESQUE}

Service de l'enseignement de la gestion des ressources humaines École des Hautes Études

Commerciales

3000, chemin de la Côte-Sainte-

Catherine

Montréal (Québec), Canada

H3T 2A7

\section{Gregor MURRAY}

Département des relations

industrielles

Université Laval

Sainte-Foy (Québec), Canada

G1K 7P4
Stéphane LE QUEUX
Industrial Relations and Human
Resource Management Group
School of Management
University of New Castle
Callaghan NSW, Australia 2308 


\section{RÉSUMÉ}

Deux hypothèses sur l'affaiblissement des identités syndicales sont soumises à une épreuve empirique à partir d'une étude auprès des syndiqués d'une centrale syndicale au Québec (la Confédération des syndicats nationaux). La première insiste sur la différenciation sociale; la seconde sur l'effet structurant des conditions matérielles de travail et des stratégies des acteurs. Deux dimensions de l'identité syndicale sont étudiées : l'évaluation du caractère essentiel du syndicalisme et l'adhésion aux modalités d'action les plus caractéristiques du syndicalisme industriel. Les diverses sources de différenciation sociale exercent peu d'impact sur le caractère essentiel mais touchent lourdement l'adhésion aux modalités d'action. À l'encontre des conditions matérielles de travail, les stratégies des acteurs sont très importantes pour les deux dimensions. Les sources de différenciation sociale ne constituent pas une rupture par rapport au syndicalisme mais elles font appel à un renouveau de ses modalités d'action. Ce renouveau doit partir d'une participation des personnes porteuses de ces nouveaux intérêts dans l'élaboration de nouvelles modalités d'action collectives.

\section{SUMMARY}

Two hypotheses on the weakening of trade union identities are tested empirically by means of a study involving unionized workers of a Quebec trade union federation (Confederation of National Trade Unions). The main focus of the first hypothesis is social differentiation, of the second the structuring effect of the material working conditions and the strategies of the various actors. Two dimensions of union identity are studied: the evaluation of the essential character of unionism and the adhesion to the most characteristic action plans of industrial unionism. The diverse sources of social differentiation have little impact on the essential nature but impact heavily on adhesion to action plans. Unlike material working conditions, actor strategies are very important in both dimensions. Sources of social differentiation do not constitute a break as it relates to trade unions, but they call for a renewal in action plans. This renewal must stem from the participation of persons having these new interests in developing new collective action plans.

\section{RESUMEN}

Dos hipótesis sobre el debilitamiento de las identidades sindicales son sometidas a una experiencia empírica a partir de un estudio sobre los trabajadores sindicalizados de una central sindical del Quebec (la Confederación de los sindicatos nacionales). La primera insiste sobre la diferenciación social; la segunda sobre el efecto estructurante de las condiciones materiales de trabajo y de las estrategias de los actores. Dos dimensiones de la identidad sindical son estudiadas: la evaluación del carácter esencial del sindicalismo y la adhesión a las modalidades de acción. Contrariamente a las condiciones materiales de trabajo, las estrategias de los actores son muy importantes para las dos dimensiones. Las fuentes de diferenciación social no constituyen una ruptura con el sindicalismo sino que ellas llaman a una renovación de las modalidades de acción. Esta renovación debe partir desde una participación de las personas que encarnan esos nuevos intereses en la elaboración de nuevas modalidades de acción colectivas.

\section{BIBLIOGRAPHIE}

BARLING, Julian, Clive FULLAGAR et E. Kevin KELlOWAY (1992), The Union and its Members : A Psychological Approach, New York, Oxford University Press.

BERGERON, Jean-Guy (1994), « Les facteurs explicatifs de la propension à se syndiquer dans les services privés », Relations industrielles / Industrial Relations, vol. 49, $\mathrm{n}^{\mathrm{O}} 4$, pp. 776-793.

BRASSARD, Sylvie et Gregor MURRAY (1997), «La syndicalisation des services privés: défis pour les structures syndicales », L'écriteau, vol. 5, no 1 , pp. 10-12.

BRISKIN, Linda et Pat McDERMOTT (1993), Women Challenging Unions : Feminism, Democracy and Militancy, Toronto, University of Toronto Press.

BUREAU INTERNATIONAL DU TRAVAIL (1997), Le Travail dans le monde : relations professionnelles, démocratie et cohésion sociale, Genève, BIT.

DION, Gérard et Gérard HÉBERT (1989), «L'avenir du syndicalisme au Canada », Relations industrielles / Industrial Relations, vol. 44, $\mathrm{n}^{\mathrm{O}}$ 1, pp. 5-24.

DUBAR, Claude (1991), La Socialisation. Construction des identités sociales et professionnelles, Paris, Armand Collin.

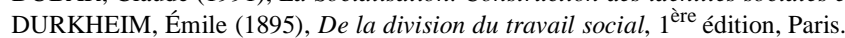

EDWARDS, Paul (1986), Conflict at Work, Oxford, Basil Blackwell.

FIORITO, Jack, Daniel G. GALLAGER et Cynthia V. FUKAMI (1988), «Satisfaction with Union Representation», Industrial and Labor Relations Review, vol. 41, $\mathrm{n}^{\circ}$ 2, pp. 294-307.

GALLIE, Duncan (1996), «Trade Union Allegiance and Decline in British Urban Labour Markets », (sous la dir. de D. GALLIE, R. PENN et M. ROSE), Trade Unionism in Recession, Oxford, Oxford University Press, pp. 140-174.

GORDON, Michael E. et coll. (1980), «Commitment to the Union : Development of a Measure and an Examination of Its Correlates », Journal of Applied Psychology Monograph, vol. 65, $\mathrm{n}^{\mathrm{o}} 4$, pp. 479-499.

GUEST, David E. et Philip DEWE (1988), «Why Do Workers Belong to Trade Unions ? », British Journal of Industrial Relations, vol. XXVI, $\mathrm{n}^{\mathrm{O}} 2$ 2, pp. 178-193.

HECKSCHER, Charles C. (1988), The New Unionism : Employee Involvement in the Changing Corporation, New York, Basic Books Inc.

HOBSBAWN, Eric (1994), Age of Extremes : The Short Twentieth Century 1914-1991, Londres, Abacus. 
HYMAN, Richard (1994), « Changing Trade Union Identities and Strategies », (sous la dir. de) R. HYMAN et A. FERNER), New Frontiers in European Industrial Relations, Oxford, Basil Blackwell, pp. 108-139.

HYMAN, Richard (1997a), «La géométrie du syndicalisme : une analyse comparative des identités et des idéologies », Relations industrielles / Industrial Relations, vol. 52, $\mathrm{n}^{\circ} 1, \mathrm{pp} .7-38$

HYMAN, Richard (1997b), «Trade Unions and Interest Representation in the Context of Globalisation », Transfer, vol. 3, $\mathrm{n}^{\mathrm{O}} 3$, pp. 515-533.

JENSON, Jane, Elizabeth HAGEN et Ceallaigh REDDY (dir.) (1988), Feminization of the Labor Force: Paradoxes and Promises, New York, Oxford University Press.

KERN, Horst et Charles F. SABEL (1992), «Trade Unions and Decentralized Production : A Sketch of Strategic Problems in the German Labour Movement» (sous la dir. de) M. REGINI, The Future of Labour Movements, Londres, Sage, pp. 217-249.

KLANDERMANS, Bert (1989), «Union Commitment : Replications and Tests in the Dutch Context », Journal of Applied Psychology, vol. 74, n⿳0 6, pp. 869-875.

LEISINK, Peter, Jim VAN LEEMPUT et Jacques VILROKX (1996), The Challenges to Trade Union in Europe. Innovation or Adaptation, Cheltenham, Edward Elgar Publishing Limited.

LE QUEUX, Stéphane (1997), Syndicalisme industriel et identités syndicales : la régulation de l'emploi comme enjeu de solidarité pour la CSN dans le cadre de la restructuration du réseau public de la santé et des services sociaux au Québec, thèse de doctorat, Département des relations industrielles, université Laval.

MADSEN, Morten (1996), « Trade Union Democracy and Individualisation : The Cases of Denmark and Sweden », Industrial Relations Journal, vol. 27, $\mathrm{n}^{\mathrm{O}} 2$, pp. 115-128.

MÜLLER-JENTSCH, Walter (1988), «Industrial Relations Theory and Trade Union Strategy », International Journal of Comparative Labour Law and Industrial Relations, vol. 4, no 3, pp. 177-190.

MURRAY, Gregor (1994), «La représentation en relations industrielles : perspective et prospective », (sous la dir. de) M AUDET et coll., La Représentation : miroir ou mirage de la démocratie au travail ?, Québec, Presses de l'université Laval, pp. 7-30.

MURRAY, Gregor (1998), « Steeling for Change : Organization and Organizing in Two USWA Districts in Canada », (sous la dir. de) K. BRONFENBRENNER et coll., Organizing to Win. New Research on Union Strategies, Ithaca et Londres, ILR Press, pp. 320-338.

NEWTON, Lucy A. et Lynn McFARLANE SHORE (1992), « A Model of Union Membership : Instrumentality, Commitment, and Opposition », Academy of Management Review, vol. 17, $\mathrm{n}^{\circ}$ 2, pp. 275-298.

OFFE, Claus et Helmut WIESENTHAL (1980), « Two Logics of Collective Action : Theoretical Notes on Social Class and Organizational Form », (sous la dir. de) M. ZEITLIN, Political Power and Social Theory, $\mathrm{n}^{\circ}$ 1, pp. 67-115.

PAQUET, Renaud (1996), «Précarité et vie syndicale », (sous la dir. de) A. GILES, A. E. SMITH et G. TRUDEAU, La mondialisation de l'économie et le travailleur, sélection de textes du XXXII ${ }^{\mathrm{e}}$ Congrès de l'ACRI, Québec, ACRI, pp. 245-256.

PERLMAN, Selig (1949), A Theory of the Labor Movement, New York, Kelley.

REYNAUD, Jean-Daniel (1989), Les Règles du jeu. L'Action collective et la régulation sociale, Paris, Armand Colin.

SAINSAULIEU, Renaud (1977), L'Identité au travail, Paris, Presses de la FNSP.

SCHETAGNE, Sylvain (1997), Le Niveau de participation des salariés à la vie syndicale et leur statut d'emploi : le cas des syndicats du secteur des services privés affiliés à la CSN, mémoire de maîtrise, Département des relations industrielles, université Laval.

SINCLAIR, Diane M. (1996), « The Importance of Gender for Participation in and Attitudes to Trade Unionism », Industrial Relations Journal, vol. 27, $\mathrm{n}^{\mathrm{O}} 3$, pp. 239-252.

TIXIER, Pierre-Éric (1992), Mutation ou déclin du syndicalisme ? : le cas de la CFDT, Paris, PUF.

VALKENBURG, Ben (1996), «Individualization and Solidarity: The Challenge of Modernization », (sous la dir. de) P. LEISINK, J. VAN LEEMPUT et J. VILROKX, The Challenges to Trade Unions in Europe: Innovation or Adaptation?, Cheltenham, Edward Elgar Publishing Limited, pp. 89-104.

WHEELER, Hoyt N. et John A. McCLENDON (1991), «The Individual Decision to Unionize », (sous la dir. de) G. STRAUSS, D. G. GALLAGHER et J. FIORITO, The State of the Unions, Madison, IRRA, pp. 47-84. 